\title{
Antiguided Ribbon Laser Concept
}

R. Beach, M. Feit, R. Page, L. Brasure, R. Wilcox, S. Payne

\section{December 13, 2000}

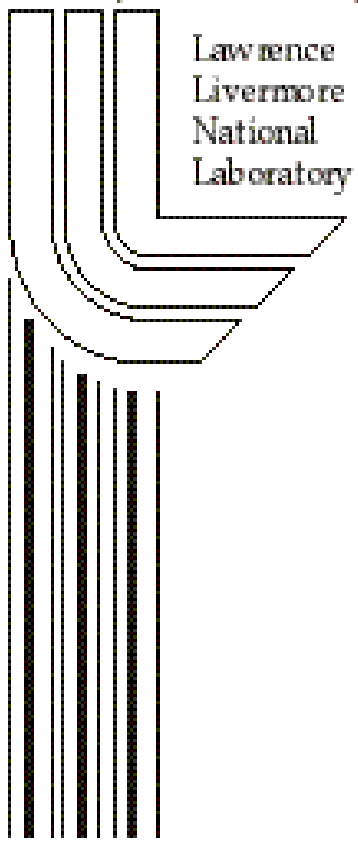




\section{DISCLAIMER}

This document was prepared as an account of work sponsored by an agency of the United States Government. Neither the United States Government nor the University of California nor any of their employees, makes any warranty, express or implied, or assumes any legal liability or responsibility for the accuracy, completeness, or usefulness of any information, apparatus, product, or process disclosed, or represents that its use would not infringe privately owned rights. Reference herein to any specific commercial product, process, or service by trade name, trademark, manufacturer, or otherwise, does not necessarily constitute or imply its endorsement, recommendation, or favoring by the United States Government or the University of California. The views and opinions of authors expressed herein do not necessarily state or reflect those of the United States Government or the University of California, and shall not be used for advertising or product endorsement purposes.

This work was performed under the auspices of the U. S. Department of Energy by the University of California, Lawrence Livermore National Laboratory under Contract No. W-7405-Eng-48.

This report has been reproduced directly from the best available copy.

Available electronically at http://www.doc.gov/bridge

Available for a processing fee to U.S. Department of Energy

And its contractors in paper from

U.S. Department of Energy

Office of Scientific and Technical Information

P.O. Box 62

Oak Ridge, TN 37831-0062

Telephone: (865) 576-8401

Facsimile: (865) 576-5728

E-mail: reports@adonis.osti.gov

Available for the sale to the public from

U.S. Department of Commerce

National Technical Information Service

5285 Port Royal Road

Springfield, VA 22161

Telephone: (800) 553-6847

Facsimile: (703) 605-6900

E-mail: orders@ntis.fedworld.gov

Online ordering: http://www.ntis.gov/ordering.htm

OR

Lawrence Livermore National Laboratory

Technical Information Department's Digital Library

http:/ / www.llnl.gov/tid/Library.html 


\title{
Antiguided Ribbon Laser Concept
}

\author{
Interim Report to Craig Denman, AFRL \\ Ray Beach, Mike Feit, Ralph Page, LeAnn Brasure, Russell Wilcox and Steve Payne \\ Lawrence Livermore National Laboratory \\ Livermore, California 94550 \\ December 13, 2000
}

\section{Introduction}

We propose a new, robustly scalable technique for phase locking multiple gain cores in a fiber structure based on antiguiding or radiative coupling, rather than the more commonly pursued method of evanescent wave phase locking. Our focus is on a ribbonlike geometry in which a waveguide region contains multiple gain cores arranged in a periodic array. The distinguishing feature of such antiguiding structures is that refractive index of the gain cores is lower than or equal to that of the surrounding waveguide regions. This is just the opposite of evanescently phase locked structures in which the gain cores have higher refractive index than the surrounding regions. The critical design considerations in the structures proposed within are: first that they strongly favor oscillation in a single transverse mode, and second that this strongly favored mode exhibits good intensity uniformity across the entire array of gain cores. We require single mode operation so that a static phase corrector placed in the near field of the ribbon laser's output can optimize the phase across the aperture to achieve a high Strehl ratio in the far field. The requirement that the strongly favored mode exhibit good uniformity across the entire array of gain cores is necessary to ensure that the ribbon structure's gain saturates in a uniform manner, so as not to increase the propensity of the device to operate in multiple transverse modes. Taken together, these two design considerations lead to the surprising result that optimized structures have equal refractive indices in their gain cores and the no-gain surrounding waveguide regions.

Because we are considering ribbon structures, a one-dimensional transverse treatment provides a reasonable model for the properties of these devices. To facilitate our analysis we develop and present a simple but elegant technique using electric field propagators to generate the eigenmode spectrum of the ribbon structures. Afterwards, we execute a full two-dimensional transverse analysis to accurately assess modal gain discrimination. Interestingly, by deriving the eigenmode spectrum of ribbon structures we are able to make the connection with photonic (so-called "holey") crystal fibers, which are constructed with actual physical holes in the glass. ${ }^{1}$ Our calculations reveal similar formation of bandgaps in the allowed wavevector values for ribbon fibers with a periodic modulation in the refractive index, as typified by holey fibers.

Because the ribbon structures proposed here contain a waveguiding region that is embedded in a lower index outer cladding region, they have many features in common with single-core double-clad fiber lasers. The development of these double-clad fiber lasers has brought fiber lasers to the forefront of possible approaches for high beam quality, high average power continuous-wave fiber laser sources. However, one of the draw backs of the single-core double-clad fiber laser approach for generating high average power is that the pump light is 
delivered into the end of the fiber, which requires diodes with radiance conditioned outputs. Our proposed pumping approach benefits from the planar ribbon structure, since it is compatible with the use of laser diode bars without radiance conditioning for pump excitation.

Because individual fiber cores are believed to be limited to roughly $100 \mathrm{~W}$ of average power generation ${ }^{2}$ due to the output facet damage limit, very high average power fiber systems are anticipated to require phase combining many individual apertures. The most commonly used approach for phase locking multiple apertures or gain cores together is the use evanescent wave coupling to phase lock the cores to their nearest neighbors. One such layout entails evanescent phase locking in which multiple gain cores are distributed around the perimeter of a larger pump-cladding region in a circular layout $^{3}$. In this approach, the evanescent wave from each core is adjusted to overlap its neighboring cores following the same technique that was used in early diode bar development to phase lock multiple stripe devices ${ }^{4}$. To date, the results from this type of evanescent nearest neighbor phase locking have been inconclusive. Another arrangement is based on hexagonal packing of the cores, and has apparently offered impressive initial results for a seven-core case ${ }^{5}$. However, with evanescent phase locking only nearest neighbors communicate with one another so that when coupling many cores together, it is anticipated that a general degradation of the phase fidelity for cores will occur, the farther away from each other they are.

The ribbon structure proposed here contains multiple gain-loaded cores in a linear array, with a non-evanescent approach to keep the cores' output radiation coherently phased together. This five-core prototype ribbon fiber is shown schematically in Fig. 1. In this approach, the gain elements are "antiguided" in a "leaky waveguide" array, analogous to the most successful scheme for phasing laser diode elements ${ }^{6}$. The antiguided cores are arranged in a row, and the cores are situated in a long aspect ratio rectangle or "ribbon" with a slightly higher index than the outer pump-cladding medium. The ribbon is encapsulated in a cladding that guides the pump light, analogous to current single-core cladding-pumped fibers. Scaling consists of increasing the number of antiguided cores. We believe this scheme will scale to higher power than evanescently coupled cores because of the strong phase locking inherent in the approach.

To explore key aspects of our proposed ribbon fiber, we start by investigating a 5core device. Although the ribbon structure is unusual, similarly complex fiber structures have been made in silica, including more complex annular arrays of doped and undoped material, evanescently coupled cores, and a variety of odd-shaped cladding-pumped fibers, bundles, and close-packed matrices. This can be accomplished using preform packing and pulling technologies. Means of drawing fibers such that the shape of the preform is preserved have been demonstrated and commercialized, ${ }^{7}$ so the resulting ribbon reflects the spatial characteristics of the preform. 


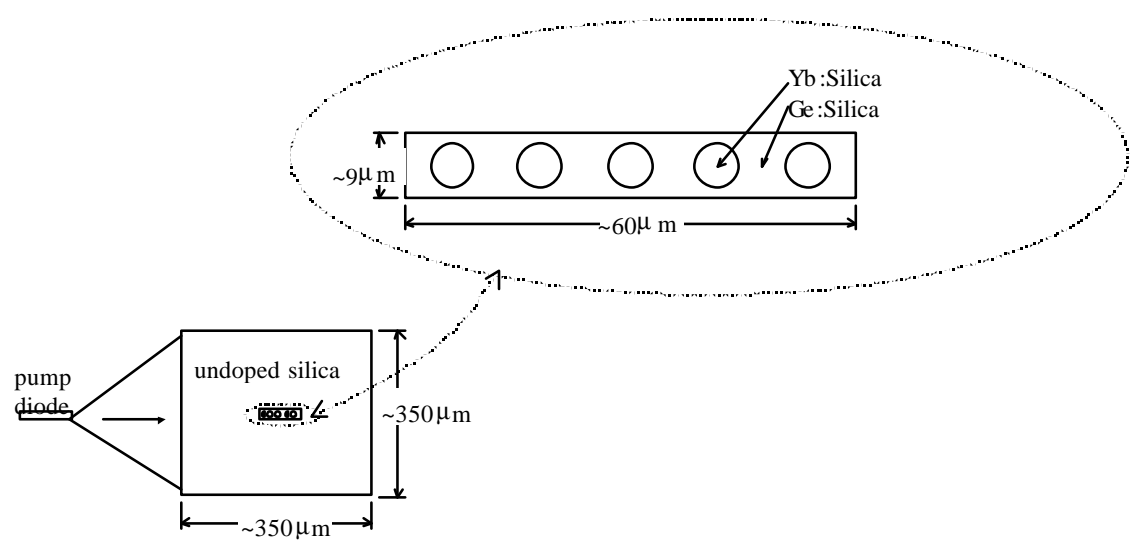

Figure 1 Cross-sectional view of 5-core ribbon fiber.

To be specific with regard to refractive index values, we assume that the structure is fabricated out of pure fused silica along with germanium and fluorine dopants to control the refractive index, and $\mathrm{Yb}$ or $\mathrm{Nd}$ doping to provide the gain-loaded regions. The round doped core sections serve as the gain regions for the optical wave that is confined to the $\sim 9 \mu \mathrm{m}$ high by $\sim 60 \mu \mathrm{m}$ wide rectangular waveguide region. This rectangular region may be doped with $\mathrm{Ge}$, which increases the index of the material surrounding the doped cores in a specified manner. The pump radiation is confined to the larger $\sim 350 \mu \mathrm{m}$ by $\sim 350 \mu \mathrm{m}$ square region, which we here assume is pure silica and so has a lower refractive index than the waveguide region.

\section{One-Dimensional Theory with Periodic Refractive Index and Gain}

To motivate our results, we begin by analyzing the simpler problem of the onedimensional structure that is related to the two-dimensional structure of Fig. 1. We take a lineout of the index profile along the centerline of the Fig. 1 structure, and plot in Fig. 2 a particular refractive index profile to serve as an illustrative case. To further simplify our analysis we will also assume the electric field polarization corresponds to a TE wave. 


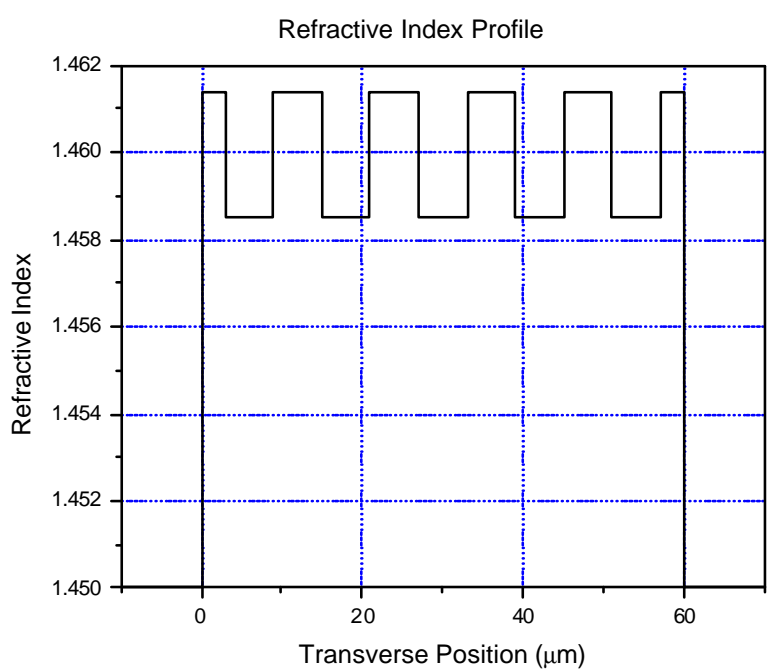

(a)

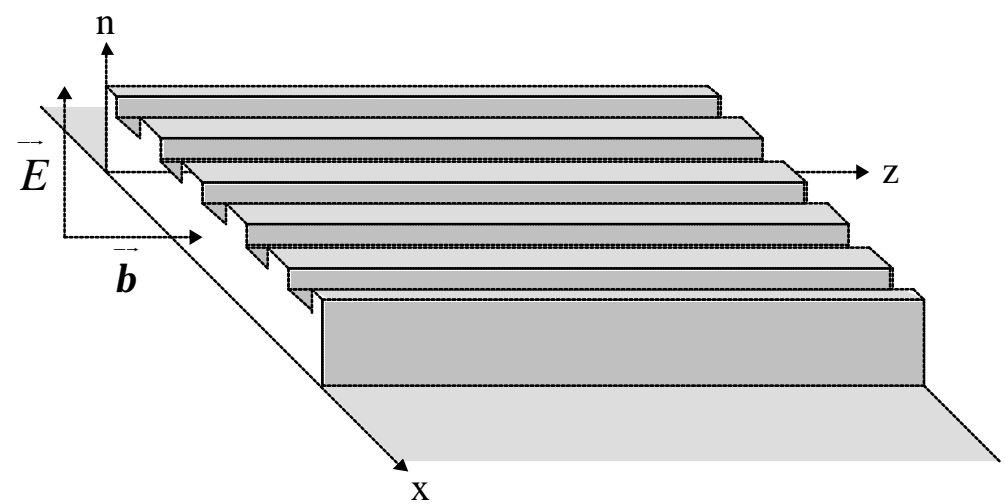

(b)

Figure 2 (a) Refractive index profile as a function of transverse position in the one-dimensional approximation to our proposed ribbon waveguide structure given in Fig. 1. The gain regions coincide with the lower index segments in the waveguide region. (b) Coordinate system used in our analysis for the TE polarized wave (w.r.t. refractive index boundaries) as shown.

We begin with the wave equation for the electric field in one of the constant index strips along the waveguide,

$$
\left(\nabla^{2}-\left(\frac{n}{c}\right)^{2} \partial_{t}^{2}\right) \vec{\varepsilon}(\vec{r}, t)=0 .
$$

Restricting the electric field to be TE polarized (the electric field parallel to the interfaces between the index segments that make up the ribbon), we write the electric field in terms of its frequency, $\omega$, and longitudinal k-vector, $\beta$, as,

$\vec{\varepsilon}(\vec{r}, t)=\widehat{u_{y}} E(x) e^{i(\omega t-\beta z)}$,

where $\widehat{u_{y}}$ is a unit vector in the y-direction. Substituting (2) into (1), then gives the equation that must be satisfied by $\mathrm{E}(\mathrm{x})$,

$\frac{d^{2} E(x)}{d x^{2}}=-\left(\left(\frac{n \omega}{c}\right)^{2}-\beta^{2}\right) E(x)$,

which is the one-dimensional Helmoholtz equation, or an eigenvalue equation for the Laplacian. Due to the polarization direction of the electric field (TE wave), the boundary condition to be satisfied at the interfaces between neighboring strips having differing refractive indices in Fig. 2 is one of continuity, i.e., the electric field amplitudes are the 
same on each side of the boundary. In addition to the continuity of the electric field amplitude at the interfaces in the ribbon structure, the second order differential equation (3) for the electric field amplitude imposes a continuity condition on $\mathrm{dE} / \mathrm{dx}$ at the interfaces. If $\mathrm{dE} / \mathrm{dx}$ where not continuous at the interfaces, then $\mathrm{d}^{2} \mathrm{E} / \mathrm{dx}^{2}$ would blow up at those locations leading to infinitely large values of the electric field. Finally, the boundary condition on the electric field amplitude outside of the rectangular waveguiding structure of Fig. 2 is that it approaches zero at large distances from the waveguide. Summarizing, the boundary conditions to be satisfied by the electric field are,

$$
\begin{aligned}
& E\left(x_{i}^{-}\right)=E\left(x_{i}^{+}\right), \\
& \frac{d E}{d x}\left|x=x_{i}^{-}=\frac{d E}{d x}\right| x=x_{i}^{+}, \\
& E(x) \rightarrow 0 \text { as } x \rightarrow \pm \infty,
\end{aligned}
$$

where $x_{i}^{-}$and $x_{i}^{+}$refer to the limiting values of $x$ at the $i^{\text {th }}$ interface when approached from the negative and positive sides, respectively. Because we are specifically interested in ensuring we develop designs that will preferentially support only a single transverse mode, we must find all electric field eigenmode solutions to (3) that satisfy the boundary conditions (4) through (6). These eigenmode solutions will be defined in terms of their longitudinal $\mathrm{k}$-vector values, $\beta$.

Our method of solution as outlined here will be to arbitrarily define an electric field amplitude of unit intensity at $x=0$ for the structure in Fig. 2, and then assume a kvector value. To determine whether the assumed $\mathrm{k}$-vector value corresponds to an actual eigenmode of the structure, we will then propagate the electric field across the structure from $x=0$ to well beyond the waveguiding portion of the structure (past $60 \mu \mathrm{m}$ in Fig. 2). Applying boundary condition (6) then demands that if the assumed k-vector value corresponds to an actual eigenmode, the electric field amplitude will approach 0 as $\mathrm{X}$ increases without bound.

The propagation of the electric field across the structure can be carried out numerically using (3) to incrementally step $\mathrm{E}$ and $\mathrm{dE} / \mathrm{dx}$ across the structure given initial values for both quantities at $\mathrm{x}=0$. However, a quicker method and the one we use here takes advantage of analytic propagators to propagate the field across an entire constant index segment of the structure in a single step. The advantage of the analytic propagator method, which we outline below, is that it is extremely efficient and applicable to very large structures (hundreds of cores) that would bog down the calculation using the more straightforward numerical incremental step calculation.

To begin the calculation, we arbitrarily choose a target k-vector value for $\beta$ in (3) and define both $\mathrm{E}$ and $\mathrm{dE} / \mathrm{dx}$ at $\mathrm{x}=0^{-}$in Fig. 2. The value of $\beta$ is not completely arbitrary due to the boundary condition (6). Since we require $\mathrm{E}(\mathrm{x})$ approach 0 as $\mathrm{x}$ approaches $-\infty$ we must choose $\beta>n_{\text {clad }} \omega / c$, where $n_{\text {clad }}$ is the refractive index value in the cladding region $x<0$ or $x>60 \mu \mathrm{m}$ in Fig. 2. As already mentioned, we can arbitrarily set the value 
of $\mathrm{E}\left(\mathrm{x}=0^{-}\right)=1$, however the value of $\mathrm{dE} /\left.\mathrm{dx}\right|_{\mathrm{x}=0^{-}}$is not arbitrary. The functional form of $\mathrm{E}(\mathrm{x})$ for $\mathrm{x}<0$ is

$E(x)=e^{\sqrt{\beta^{2}-\left(\frac{n_{l} \omega}{c}\right)^{2}} x}$,

which decays exponentially to 0 as $\mathrm{x} \rightarrow-\infty$. With $\mathrm{E}(\mathrm{x})$ given by (7), the value of $\mathrm{dE} /\left.\mathrm{dx}\right|_{\mathrm{x}=0^{-}}$is given by,

$\left.\frac{d E}{d x}\right|_{x=0^{-}}=\sqrt{\beta^{2}-\left(\frac{n_{l} \omega}{c}\right)^{2}}$.

Denoting the refractive index of the $\mathrm{i}^{\text {th }}$ constant index segment of the waveguide structure described in Fig. 2 by $n_{i}$, the electric field and its first derivative in that segment will be given by,

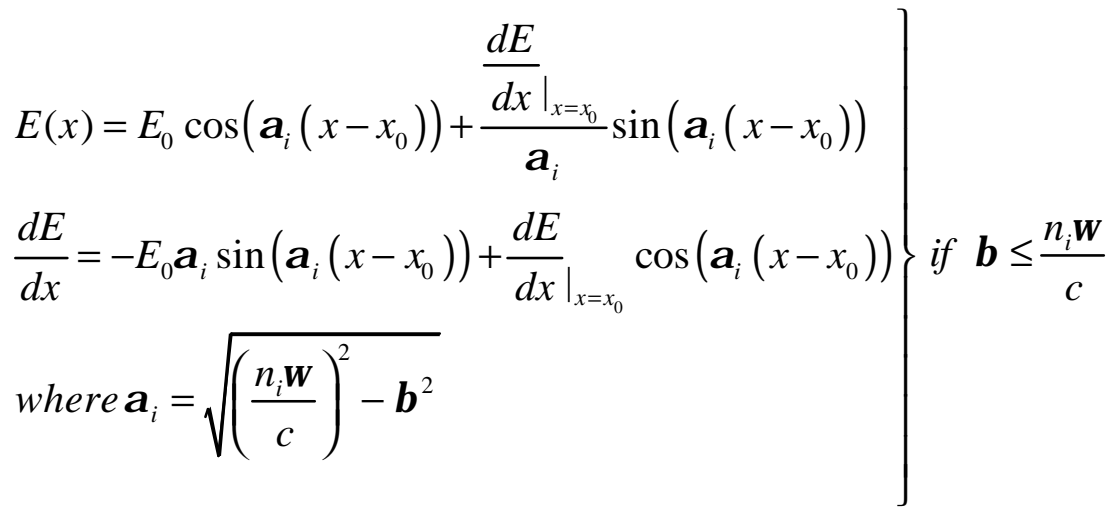

or

$\left.\begin{array}{l}E(x)=E_{0} \cosh \left(\tilde{\alpha}_{i}\left(x-x_{0}\right)\right)+\frac{\left.\frac{d E}{d x}\right|_{x=x_{0}}}{\widetilde{\alpha}_{i}} \sinh \left(\tilde{\alpha}_{i}\left(x-x_{0}\right)\right) \\ \left.\frac{d E}{d x}=E_{0} \tilde{\alpha}_{i} \sinh \left(\tilde{\alpha}_{i}\left(x-x_{0}\right)\right)+\left.\frac{d E}{d x}\right|_{x=x_{0}} \cosh \left(\tilde{\alpha}_{i}\left(x-x_{0}\right)\right)\right) \text { if } \beta>\frac{n_{i} \omega}{c}, \\ \text { where } \tilde{\alpha}_{i}=\sqrt{\beta^{2}-\left(\frac{n_{i} \omega}{c}\right)^{2}}\end{array}\right\}$

where $\mathrm{x}_{0}$ is the $\mathrm{x}$-coordinate of the far left-hand side of the segment and $\mathrm{E}_{0}$ and $\mathrm{dE} /\left.\mathrm{dx}\right|_{\mathrm{x}=\mathrm{x} 0}$ are the field and field derivative values there. Writing the field propagators as in (9) and (10) facilitates a quick calculational technique for propagating the electric field across the structure in terms of its field amplitude and the derivative of field amplitude at some initial point such as at $\mathrm{x}=0$ as given in (7) and (8). Starting with the electric field amplitude and its first derivative at the left hand side of one of the constant index 
segments, the electric field amplitude and its derivative at the right hand side of the constant index segment is given by,

$$
\left.\begin{array}{l}
E_{r h s}=E_{l h s} \cos \left(\alpha_{i} l_{i}\right)+\frac{\left.\frac{d E}{d x}\right|_{l h s}}{\alpha_{i}} \sin \left(\alpha_{i} l_{i}\right) \\
\left.\frac{d E}{d x}\right|_{r h s}=-E_{l h s} \alpha_{i} \sin \left(\alpha_{i} l_{i}\right)+\left.\frac{d E}{d x}\right|_{l h s} \cos \left(\alpha_{i} l_{i}\right)
\end{array}\right\} \text { if } \beta \leq \frac{n_{i} \omega}{c}
$$

or

$$
\left.\begin{array}{l}
E_{r h s}=E_{l h s} \cosh \left(\widetilde{\alpha}_{i} l_{i}\right)+\frac{\left.\frac{d E}{d x}\right|_{l h s}}{\widetilde{\alpha}} \sinh \left(\tilde{\alpha}_{i} l_{i}\right) \\
\left.\frac{d E}{d x}\right|_{r h s}=E_{l h s} \tilde{\alpha}_{i} \sinh \left(\tilde{\alpha}_{i} l_{i}\right)+\left.\frac{d E}{d x}\right|_{l h s} \cosh \left(\widetilde{\alpha}_{i} l_{i}\right)
\end{array}\right\} \text { if } \beta>\frac{n_{i} \omega}{c} .
$$

where $l_{\mathrm{i}}$ is the width of the $i^{\text {th }}$ constant index segment in the ribbon fiber structure, and $\alpha_{i}$ and $\tilde{\alpha}_{i}$ are as define in (9) and (10), respectively. The character of the electric field solution in any given index segment is strongly dependent on the relationship between the value of $\beta$ and $n_{i} \omega / c$. In the case of $\beta<n_{i} \omega / c$, the solution is freely radiating and the field propagates across the waveguide segment with an oscillatory behavior but does not decay in amplitude. In the case of $\beta>n_{i} \omega / c$, the fields have an exponential dependence on the transverse coordinate in the waveguide segment, which is the situation one normally thinks of as evanescent coupling.

Using the transverse propagator method outlined above, trial values of the wave vector $\beta$ in (2) can be propagated across the waveguide structure. The requirement that the trial $\beta$ value correspond to an actual electric field eigenmode of the structure is that as $\mathrm{x} \rightarrow \infty$ on the right hand side of the waveguide, the electric field amplitude goes to zero. In general, a trial value for $\beta$ will generate an electric field amplitude that either diverges toward $+\infty$ or $-\infty$ as $x \rightarrow \infty$. This suggests a general method to search for electric field eigenmode $\beta$ values. If two nearby $\beta$ values can be found that generate fields that diverge in opposite directions as $\mathrm{x} \rightarrow \infty$, then by continuity there must be an intermediate $\beta$ value such that the field it generates goes asymptotically to 0 as $x \rightarrow \infty$, i.e., a $\beta$ value corresponding to an actual electric field eigenmode. An example of this method is illustrated by the field calculations displayed in Fig. 3. 


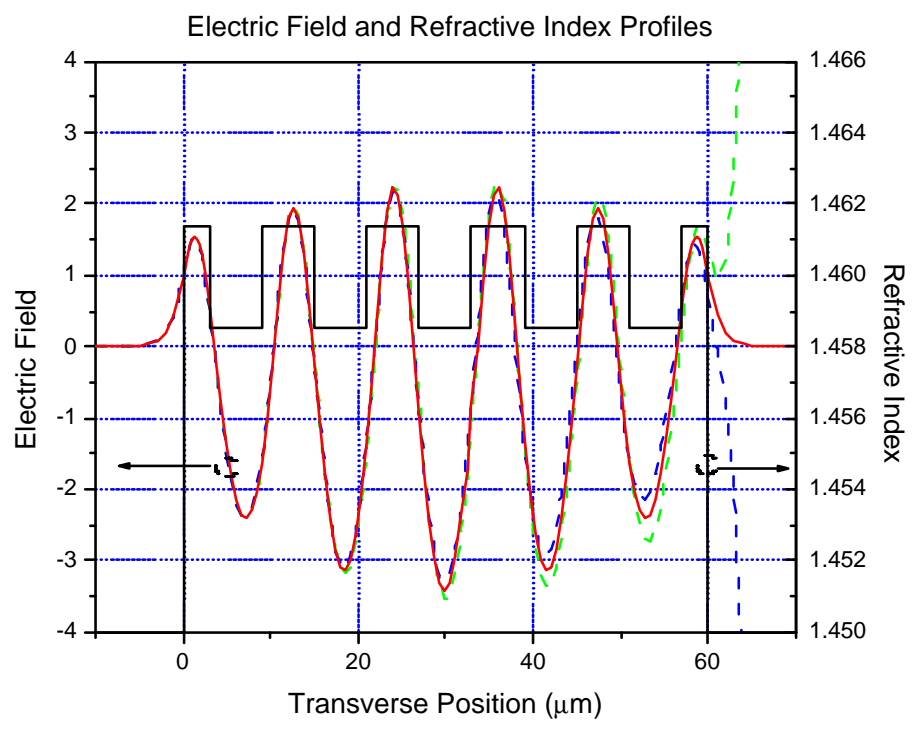

Figure 3 Electric field calculations for three different $\beta$ values for the onedimensional ribbon fiber structure described by the refractive index profile in Fig. 2. The green, blue and red lines are fields calculated using the $\beta$ values described in the text. Also shown in black is the refractive index profile.

The $\beta$ values used for the calculations in Fig. 3 are as follows:

$$
\begin{aligned}
& \beta=8.7167195 x(0.99999) \text { shown as a blue dotted line } \\
& \beta=8.7167195 \text { shown as a solid red line } \\
& \beta=8.7167195 x(1.00001) \text { shown as a green dotted line }
\end{aligned}
$$

Starting at the left-hand side of the waveguide and using the initial conditions for the electric field and its derivative given by (7) and (8), respectively, the fields are propagated to the right hand side of the waveguide. The smallest of the three $\beta$ values leads to field diverging to $-\infty$ and the largest of the $\beta$ values leads to a field diverging toward $+\infty$ as $x \rightarrow \infty$. The intermediate $\beta$ value generates a field that goes to zero and so corresponds to the $\mathrm{k}$-vector value of a true electric field eigenmode. This suggests the algorithm we will then use to find all eigenmodes of the structure. Starting with the smallest allowed $\beta$ value, calculations of the field intensity will be made for the propagated field somewhere past the right hand boundary of the waveguide. $\beta$ values will be increased incrementally and every time the diverging electric field on the right hand side of the structure is found to change sign we know by continuity that there exists a $\beta$ value corresponding to a true electric field eigenmode between the corresponding $\beta$ values for the diverging fields. Using the technique of repeated bisection, the method can then be iterated until the true beta values corresponding to eigenmodes are calculated to any desired accuracy. This technique is generally applicable to arbitrary waveguide structures and can quickly yield the entire spectrum of a given structure's allowed eigenmodes. 
Applying the above described electric field eigenmode search algorithm to the one-dimensional structure described in Fig. 2, and fixing the vacuum wavelength of the radiation at $1.05 \mu \mathrm{m}$, leads to the $\beta$ values of allowed eigenmodes detailed in Table I below. In this calculation, the refractive indices of the gain region, the no-gain region within the waveguide region, and the outermost cladding region are taken to be 1.4585 , 1.4614 , and 1.45 , respectively.

Table I Eigenmodes of 1-Dimensional Structure Shown in Fig. 2

\begin{tabular}{|c|c|c|c|}
\hline $\begin{array}{c}\text { Mode Reference } \\
\text { Number }\end{array}$ & $\beta$ (1/microns) & $\begin{array}{c}\text { Overlap } \\
\Gamma\end{array}$ & $\begin{array}{c}\text { Effective Index } \\
\mathbf{n}_{\text {eff }}\end{array}$ \\
\hline 1 & 8.73955 & 0.16998 & 1.46049 \\
\hline 2 & 8.73935 & 0.15591 & 1.46046 \\
\hline 3 & 8.73908 & 0.13437 & 1.46041 \\
\hline 4 & 8.73883 & 0.11206 & 1.46037 \\
\hline 5 & 8.73046 & 0.40377 & 1.45897 \\
\hline 6 & 8.73045 & 0.40003 & 1.45897 \\
\hline 7 & 8.72765 & 0.60593 & 1.45850 \\
\hline 8 & 8.72627 & 0.54302 & 1.45827 \\
\hline 9 & 8.72447 & 0.47906 & 1.45797 \\
\hline 10 & 8.72266 & 0.40726 & 1.45767 \\
\hline 11 & 8.71672 & 0.73056 & 1.45667 \\
\hline 12 & 8.71426 & 0.64681 & 1.45626 \\
\hline 13 & 8.71107 & 0.59410 & 1.45573 \\
\hline 14 & 8.70750 & 0.55889 & 1.45513 \\
\hline 15 & 8.70391 & 0.52744 & 1.45453 \\
\hline 16 & 8.69740 & 0.58323 & 1.45345 \\
\hline 17 & 8.69342 & 0.54614 & 1.45278 \\
\hline 18 & 8.68868 & 0.52371 & 1.45199 \\
\hline 19 & 8.68361 & 0.50114 & 1.45114 \\
\hline
\end{tabular}

To ensure the far field of the structure of the ribbon fiber can be modified in a controlled way with a static phase corrector plate placed in the near field, it is critical that the device operate in a single transverse mode. To completely answer the questions of what modes will lase in our ribbon fiber structure and with what discrimination between neighboring modes, requires a detailed wave-optics/energetics calculation that takes into account gain saturation. Here we will be satisfied with an approximate treatment in which gain saturation is ignored. The gain experienced by a different laser modes in this approximation will then be proportional to the overlap, $\Gamma$, of the mode's intensity envelope with the gain-loaded portion of the fiber,

$\Gamma=\frac{\int|E(x)|^{2} g(x) d x}{\int|E(x)|^{2} d x}$,

where $\mathrm{g}(\mathrm{x})$ is a function with value unity in those portions of the fiber that are gain loaded and 0 where there is no gain loading. A straightforward calculation once the eigenmode fields are known, these overlap values are tabulated for the various allowed 
electric field eigenmodes in Table I (recall that the gain-loaded regions correspond to the low refractive index segments). The ideal situation for ensuring single transverse mode operation is then a single mode having a large $\Gamma$ value that is well separated from the $\Gamma$ values associated with all other modes. Also listed in Table I are the effective index values, $\mathrm{n}_{\mathrm{eff}}$, associated with the various eigenmodes of the structure as defined by,

$$
\beta=\frac{n_{e f f} \omega}{c}
$$

From (14) it is seen that $\mathrm{c} / \mathrm{n}_{\mathrm{eff}}$ is just the phase velocity associated with the eigenmode as it propagates in the ribbon structure. Figure 4 plots the $\Gamma$ values against the effective index values for the eigenmodes in Table I. It is assumes that the gain-loaded areas are in the low-index regions. The plot in Fig. 4 is closely related to a plot of the complex versus real refractive index values that characterize each of the eigenmodes. The electric field that propagates down a gain loaded ribbon fiber structure shown in Fig. 3 is given by,

$\varepsilon(\vec{r}, t)=E(x) e^{i(\omega t-\beta z)} e^{\frac{1}{2} \alpha \Gamma z}$,

where $\alpha$ is the gain per unit length in the gain-loaded portion of the ribbon fiber structure. Rewriting (15) and introducing the effective index defined in (14), but extending it to complex values,

$\varepsilon(\vec{r}, t)=E(x) e^{i\left(\omega t-\left(\beta+\frac{1}{2} \alpha \Gamma\right) z\right)}=E(x) e^{i\left(\omega t-\frac{n_{\text {eff } \omega}}{c} z\right)}$,

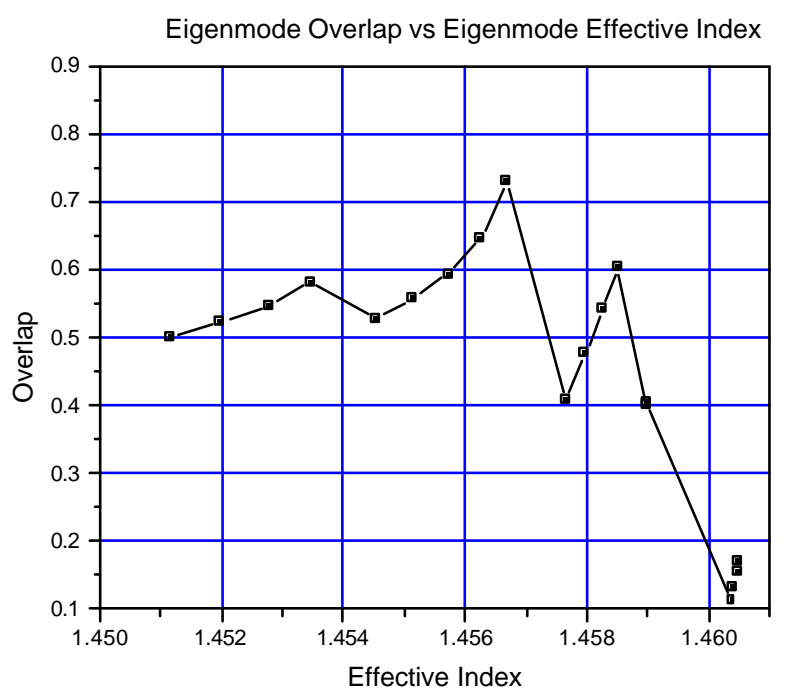

Figure 4 The eigenmode overlap with the gain region is plotted against eigenmode effective index for the for the modes listed in Table I. 
one immediately sees the connection between $\Gamma$ and the imaginary part of the complex effective refractive index,

$\operatorname{Im}\left(n_{e f f}\right)=\frac{c \alpha \Gamma}{2 \omega}$

which are proportional to each other. Going back to the plot in Fig. 4, it is seen that the $\mathrm{x}$-axis is just the real part of the complex refractive index and the $\mathrm{y}$-axis is the imaginary part of the complex refractive index up to the multiplicative factor given in (17). Starting to be evident in Fig. 4 are gaps in the allowed values of effective index. As will be discussed in more detail later, these band gaps in effective index develop in the periodic optical structures considered here for the same reasons that bandgaps in energy develop for electrons in crystalline solids. The mathematics is analogous in both cases. In essence the one-dimensional ribbon structures constitute photonic crystals in the same mathematical and physical sense as they are for the holey fibers of current intense interest.

Examining the plot in Fig. 4 and the Table I data, the mode with the highest gain is the $11^{\text {th }}$ one listed. The intensity envelope of this particular mode is shown in Fig. 5, illustrating that its high intensity lobes overlap the gain-loaded portion of the structure.

Eigenmode Intensity Profile \#11

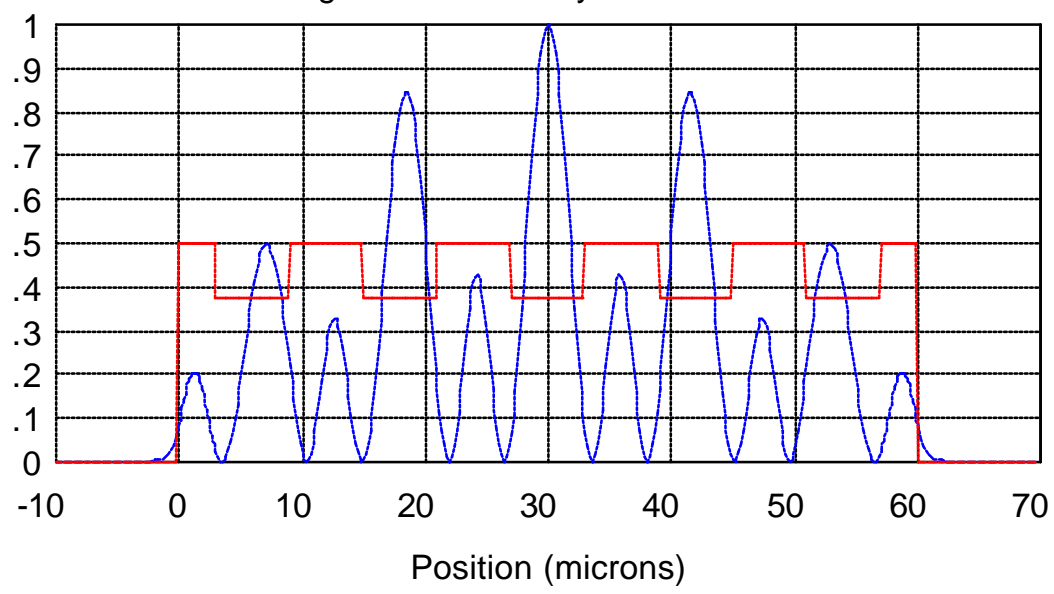

Figure 5 Intensity envelope (shown in blue) of the electric field eigenmode with the highest $\Gamma$ value for the 5-core ribbon structure detailed in Fig. 4.

Superimposed in red is the refractive index profile of the structure (not to scale). The gain is located in the lower index regions within the waveguide structure.

Although the mode shown in Fig. 5 has the highest gain, it does not have constant lobe intensity in the gain regions across the ribbon structure. The two outlying gain regions see half the peak intensity of the central gain region. Under conditions of strong gain saturation that would be required in an efficient laser, this implies the two outlying regions will not be as strongly extracted as the central regions, leaving an unbalanced gain profile across the structure. This in turn may encourage an additional mode coming 
in that extracts these outlying regions. This problem of nonuniform peak intensity in the various gain cores can be attacked by specifically designing the ribbon structure to ensure the peak intensity is unchanged from core to core.

\section{Design Criteria for Constant Mode Intensity in Gain Cores}

Here we deduce the design rules for the one-dimensional ribbon structure so that the peak intensity in each gain core is a constant. Equation (3) hints at how to proceed. If we can arrange for the electric field to null at each interface between the various index regions that make up the ribbon structure, then the lobes in the gain regions will all be identical as will the lobes in the non-gain regions. To proceed we will fix a wavevector value and then construct the ribbon structure by letting the widths of the gain and no-gain regions vary. To maintain a connection with the ribbon structure and eigenmode shown in Fig. 5, we will use the same refractive indices for the gain and no-gain regions as were used there. To begin, we will focus on a wavevector value of $8.717 / \mu \mathrm{m}$, the same wavevector value as eigenmode \#11 shown in Fig. 5. Calling the refractive index values of the gain and no-gain regions $n_{g}$ and $n_{n g}$ respectively, we adjust the widths of the gain and no-gain regions such that the following two equations are simultaneously satisfied,

$$
\begin{aligned}
& l_{g} \sqrt{\left(\frac{n_{g} \omega}{c}\right)^{2}-\beta^{2}}=\pi \\
& l_{n g} \sqrt{\left(\frac{n_{n g} \omega}{c}\right)^{2}-\beta^{2}}=\pi
\end{aligned},
$$

where $l_{\mathrm{g}}$ and $l_{\text {ng }}$ are the widths of the gain and no-gain segments, respectively. Equation (18) ensures one complete lobe will just fit into each of the index segments that makes up the ribbon structure. Staying with the same vacuum wavelength of $1.05 \mu \mathrm{m}$ for the radiation under consideration, solving the above equations gives $7.29 \mu \mathrm{m}$ for the width of the gain region and $4.49 \mu \mathrm{m}$ for the width of the no-gain regions. The remaining issue concerns the width of the two end segments, which are no-gain regions. Since the boundary condition of the outer no-gain segments is different than the interior no-gain segments, separate consideration must be given as to their widths to insure the field, which is nonzero at the outer waveguide surfaces, nulls to zero at the first interior interface. This task is easy to accomplish using the boundary condition (8) and the electric field propagator given in (11). Taken together these two equations give the electric field value at the first interface surface,

$$
E_{\substack{\text { first } \\ \text { interior } \\ \text { interface }}}=\cos \left(l_{\text {edge }} \sqrt{\left(\frac{n_{n g} \omega}{c}\right)^{2}-\beta^{2}}\right)+\frac{\sqrt{\beta^{2}-\left(\frac{n_{\text {clad }} \omega}{c}\right)^{2}}}{\sqrt{\left(\frac{n_{n g} \omega}{c}\right)^{2}-\beta^{2}}} \sin \left(l_{\text {edge }} \sqrt{\left(\frac{n_{n g} \omega}{c}\right)^{2}-\beta^{2}}\right),
$$


where $n_{\text {clad }}$ is the index of the pump cladding surrounding the waveguide region, $l_{\text {edge }}$ is the width of the no-gain edge region, and we have arbitrarily chosen the magnitude of the electric field to be unity at the left hand edge of the waveguide structure $(x=0)$. Requiring the field at the first interior interface be zero leads to the following condition to be satisfied by the edge widths,

$$
\frac{\sqrt{\left(\frac{n_{n g} \omega}{c}\right)^{2}-\beta^{2}}}{\sqrt{\beta^{2}-\left(\frac{n_{\text {clad }} \omega}{c}\right)^{2}}}+\tan \left(l_{\text {edge }} \sqrt{\left(\frac{n_{n g} \omega}{c}\right)^{2}-\beta^{2}}\right)=0 .
$$

Taking the refractive index value of the cladding to be the same as before, 1.45 , the edge length must be $3.497 \mu \mathrm{m}$ to satisfy (20). Table II summarizes the detailed design of the ribbon fiber and Fig. (6) gives a summary of this structure's eigenmode spectrum in a plot of overlap vs effective index value.

Table II Detailed Design of One -Dimensional Ribbon Fiber Structu
\begin{tabular}{|c|c|c|}
\hline Width $(\mu \mathbf{m})$ & Refractive Index & Gain Loaded? \\
\hline 10 & 1.4500 & no \\
\hline 3.49727 & 1.4614 & no \\
\hline 7.29079 & 1.4585 & yes \\
\hline 4.49309 & 1.4614 & no \\
\hline 7.29079 & 1.4585 & yes \\
\hline 4.49309 & 1.4614 & no \\
\hline 7.29079 & 1.4585 & yes \\
\hline 4.49309 & 1.4614 & no \\
\hline 7.29079 & 1.4585 & yes \\
\hline 4.49309 & 1.4614 & no \\
\hline 7.29079 & 1.4585 & yes \\
\hline 3.49727 & 1.4614 & no \\
\hline 10 & 1.4500 & no \\
\hline
\end{tabular}

For mode \#9 as shown in Fig. 7, each gain region contains a single lobe with uniform peak intensity, which is the desired result. 


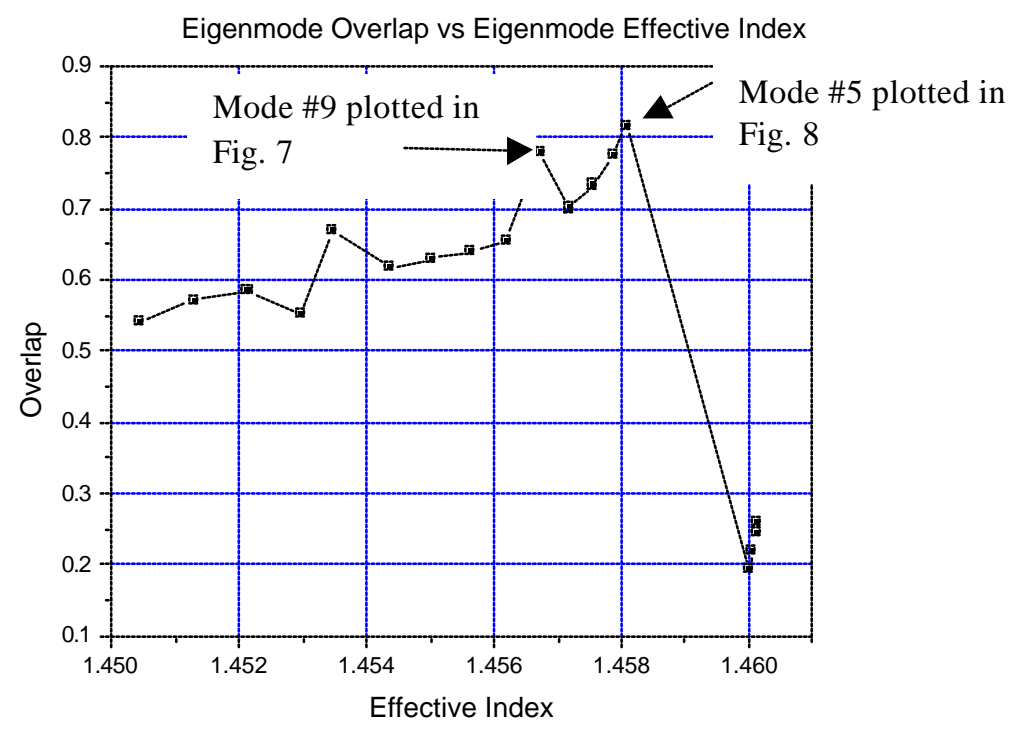

Figure 6 The eigenmode overlap with the gain region is plotted against effective index. Mode \#9 was designed to exhibit uniform peak intensities across the waveguide structure, although mode \#5 is seen to have the highest overlap.

The ribbon structure having the mode spectrum plotted in Fig. 6 was specifically designed to ensure constant peak intensity in all the gain regions for the mode corresponding to wavevector $8.717 / \mu \mathrm{m}$, the $9^{\text {th }}$ mode listed. However, we also have the additional requirement that this mode should have the highest gain overlap (by a significant margin) to ensure good mode discrimination between itself and other possible competing modes, a constraint which is no longer satisfied. It is seen that mode \#5 now has higher overlap than the mode \#9. Examining mode \#5, which is plotted in Fig. 8, it is seen that this eigenmode does not offer a uniform intensity lobe in each of the gain cores.

Eigenmode Intensity Profile \#9

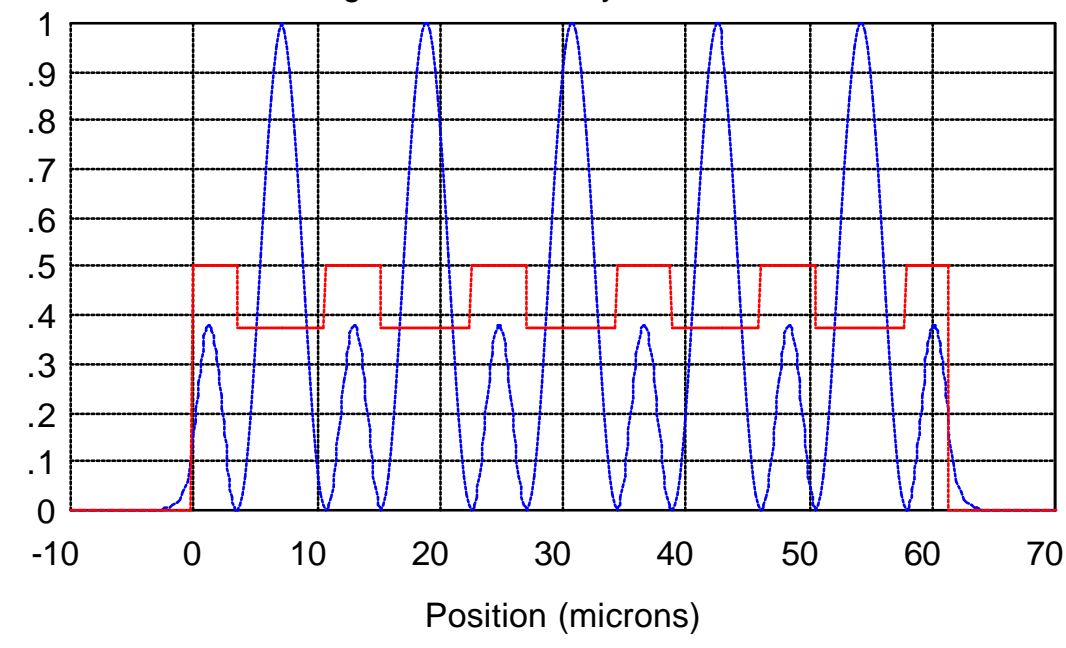

Figure 7 Intensity envelope (shown in blue) of the intensity eigenmode for which the ribbon structure was designed. The widths of the individual index segments are adjusted so that each gain region sees the same peak intensity. Superimposed in red is the refractive index profile of the structure (not to scale). The gain is located in the lower index regions within the waveguide structure. 


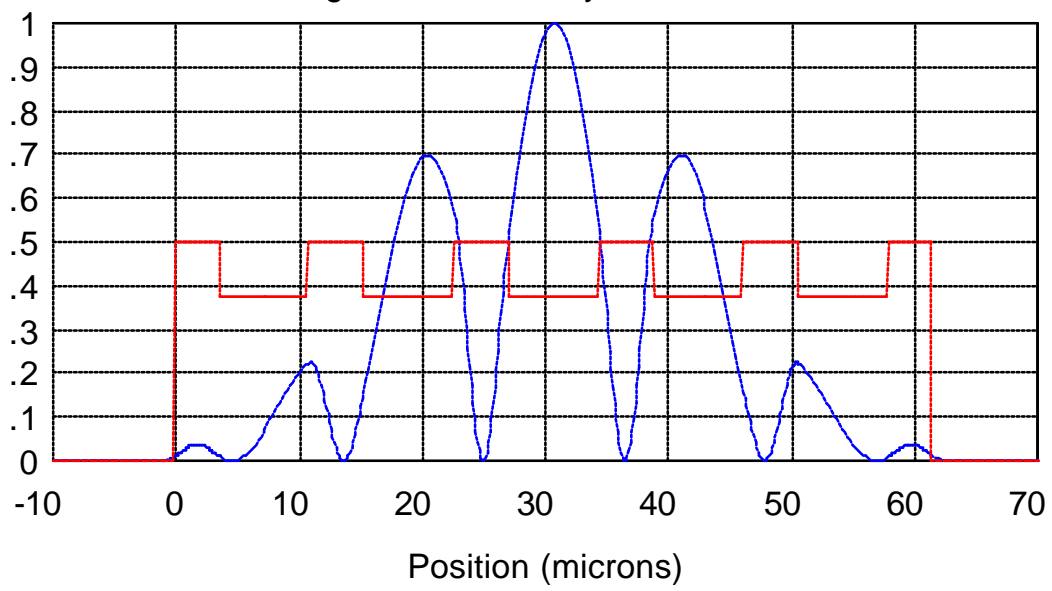

Figure 8 Mode \#5 from Fig. 6 has the highest overlap with the gain regions in the fiber structure, but does not have uniform peak intensity across the gain regions of the waveguide. Superimposed in red is the refractive index profile of the structure (not to scale).

The forgoing analysis of modes \#5 and \#9 illustrates a general difficulty that is encountered in designing an antiguiding waveguide structure for which a single mode simultaneously has the highest gain overlap and also a uniform peak intensity in the gain regions. Generally, these two conditions cannot be simultaneously satisfied in a robust way by a single transverse mode in a waveguide structure having a modulated refractive index profile.

\section{One-Dimensional Structures with Gain Variations and Constant Refractive Index}

As an alternative to the periodically modulated index structures just considered we now evaluate a waveguide structure having a uniform refractive index across its aperture and only modulate the gain profile periodically. To keep a connection with the previously analyzed case displayed in Fig. 3, we keep the outer clad index value at 1.45 and the waveguide at a constant index value of 1.4585. Furthermore we design the gain modulation profile such that an eigenmode with wavevector value $8.717 / \mu \mathrm{m}$ is the favored mode in terms of gain overlap and there are five gain cores across the aperture. We also modify the previously considered structures by now locating a gain region at the ends of the waveguiding region whereas previously there were no-gain regions at these locations (the reasons for this change will be obvious below). To begin, we arbitrarily set the gain fill factor in the interior of the waveguide structure to be 50\%. To maximize the gain overlap we wish to center the intensity peaks ( or antinodes) on the gain regions and intensity nodes on the no-gain regions, imposing a transverse gain spatial periodicity, $\ell_{\text {gain }}+\ell_{\text {no-gain }}$, given by

$\left(\ell_{\text {gain }}+\ell_{n o-\text { gain }}\right) \sqrt{\left(\frac{n_{w g} \omega}{c}\right)^{2}-\beta^{2}}=\pi$. 
Keeping the same $1.05 \mu \mathrm{m}$ vacuum wavelength as used previously, the above equation gives the gain spatial periodicity as $7.290 \mu \mathrm{m}$. The detailed design of the resulting constant index structure is given in Table III.

Table III Detailed Design of One-Dimensional Ribbon Fiber Structure With Constant Index in the Waveguide Region

\begin{tabular}{|c|c|c|}
\hline Width $(\mu \mathbf{m})$ & Refractive Index & Gain Loaded? \\
\hline 10 & 1.45 & no \\
\hline 4.36409 & 1.4585 & yes \\
\hline 3.64540 & 1.4585 & no \\
\hline 3.64540 & 1.4585 & yes \\
\hline 3.64540 & 1.4585 & no \\
\hline 3.64540 & 1.4585 & yes \\
\hline 3.64540 & 1.4585 & no \\
\hline 3.64540 & 1.4585 & yes \\
\hline 3.64540 & 1.4585 & no \\
\hline 4.36409 & 1.4585 & yes \\
\hline 10 & 1.45 & no \\
\hline
\end{tabular}

In the detailed design of the waveguide structure given in Table III, we have used an argument analogous to that used for tuning the thickness of the edge index regions of the structure referenced in Fig. 3. This argument, which led to (20), is used here to keep the gain regions centered on the intensity peaks. Performing the same type of eigenmode analysis as in the previous section leads to the eigenmode spectrum summarized in Table IV and Fig. 9, demonstrating the excellent mode discrimination that can be achieved for this ribbon structure. The waveguide was explicitly designed to optimize the overlap of mode \#5 in Table IV.

Table IV Eigenmodes of One-Dimensional Structure Summarized in Table III

\begin{tabular}{|c|c|c|c|}
\hline $\begin{array}{c}\text { Mode Reference } \\
\text { Number }\end{array}$ & $\begin{array}{c}\beta \\
(\mathbf{1} / \text { microns })\end{array}$ & $\begin{array}{c}\text { Overlap } \\
\Gamma\end{array}$ & $\begin{array}{c}\text { Effective Index } \\
\mathbf{n}_{\text {eff }}\end{array}$ \\
\hline 1 & 8.72722 & 0.50234 & 1.45843 \\
\hline 2 & 8.72593 & 0.50837 & 1.45821 \\
\hline 3 & 8.72380 & 0.51583 & 1.45786 \\
\hline 4 & 8.72082 & 0.52285 & 1.45736 \\
\hline 5 & 8.71700 & 0.84589 & 1.45672 \\
\hline 6 & 8.71235 & 0.53579 & 1.45594 \\
\hline 7 & 8.70688 & 0.54376 & 1.45503 \\
\hline 8 & 8.70063 & 0.55188 & 1.45399 \\
\hline 9 & 8.69364 & 0.55558 & 1.45282 \\
\hline 10 & 8.68602 & 0.54447 & 1.45154 \\
\hline 11 & 8.67821 & 0.46683 & 1.45024 \\
\hline
\end{tabular}




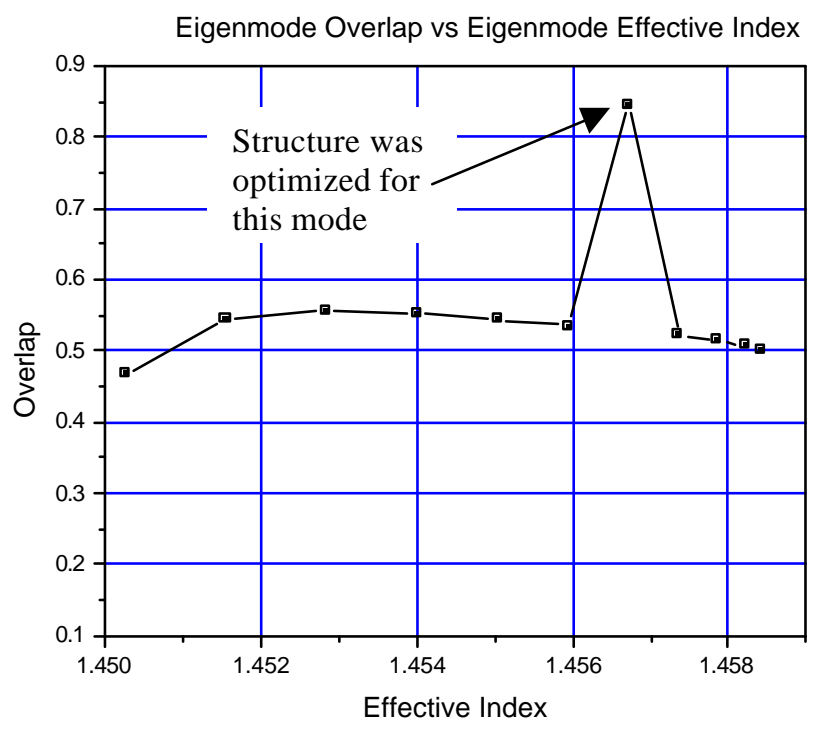

Figure 9 The eigenmode overlap with the gain region is plotted against eigenmode effective index for the modes listed in Table IV. Mode \#5, for which the structure was optimized, exhibits the highest gain overlap and has excellent mode discrimination from all other modes supported by the structure.

Figure 10 shows a plot of the intensity profile of the preferred mode (mode \#5) across the waveguide aperture. It is evident from the plot that all the gain regions will be extracted uniformly in this case, as the peak intensity of the eigenmode within each gain region is constant across the entire aperture.

Eigenmode Intensity Profile \#5

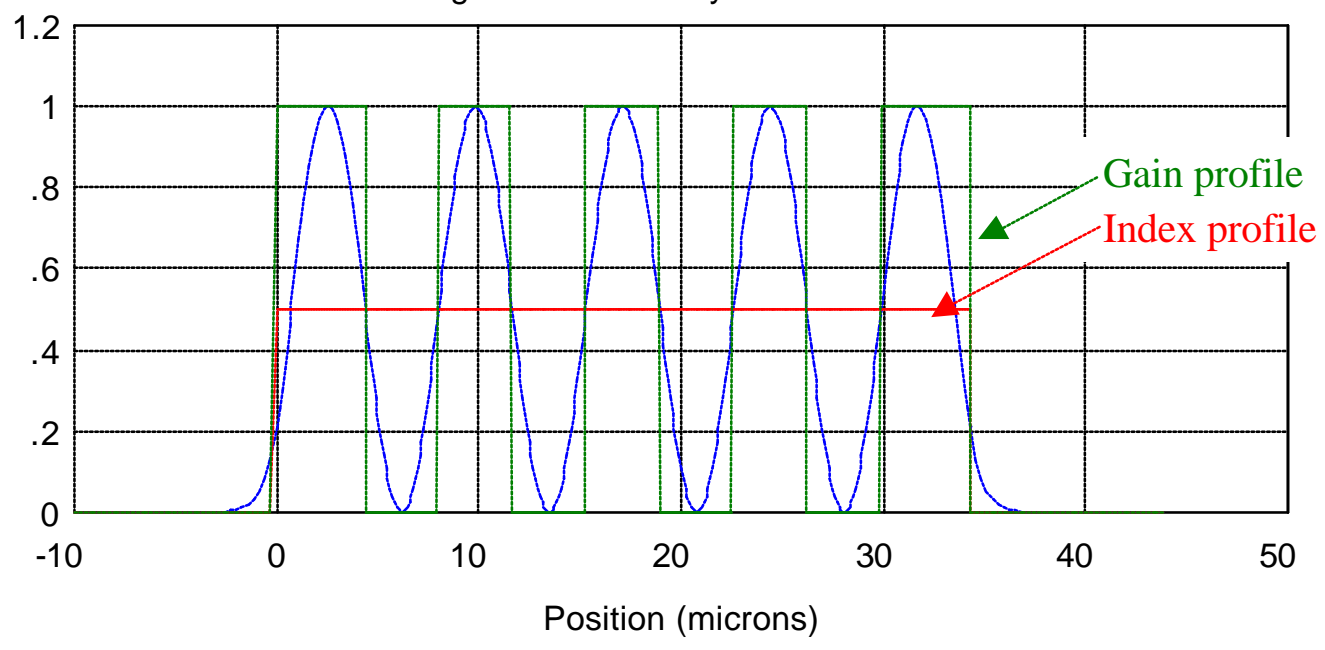

Figure 10 Plot of field intensity for mode \#5 from Table V. This mode has the highest overlap of all the allowed modes as well as a uniform peak intensity across the gain regions of the waveguide. Superimposed in green, is the gain profile of the structure with unity indicating there is gain loading and 0 indicating no gain. The waveguide index profile is indicated by the red line (not to scale) with a constant value of 1.4585 in the waveguide and 1.45 in the outer cladding region. 
The gain discrimination depicted in Fig. 9 clearly favors the single mode plotted in Fig. 10 (mode \#5). By increasing the fill factor of the gain loaded regions beyond the $50 \%$ design point considered here, the $\Gamma$ factor of the preferred mode can be increased beyond its value of 0.835 . This however will come at the expense of mode discrimination, as the $\Gamma$ factor of all modes will also increase.

As a consequence of eliminating the periodic variation in the refractive index, it is apparent that the photonic bandgap is also eliminated. This effect is akin to having a constant potential in a crystal, as is approximately evidenced by certain band states of metals (i.e. a nearly free electron model). It is also interesting to note that the highly favored mode in Fig. 10 may be regarded as the electronic wavefunction that would occur at the Brillouin zone boundary.

\section{Theory and Numerical Method for Waveguide Simulations with 2 Transverse Dimensions}

The 1-dimensional transverse theoretical model outlined above gives insight into the occurrence of desired anti-guided leaky modes in structures of the type considered here. In order to understand effects on performance of possible fabrication constraints, we have considered a number of designs with either rectangular or circular cores that either touch the boundary or are immersed in the inner cladding region. Various implementations correspond to the same 1-dimensional transverse structure (index distribution as seen on center line) analyzed in the preceding section. The optical description of such structures is not necessarily separable, except perhaps in the effective index approximation, which requires solution of many 1-dimensional transverse waveguide structures to synthesize the full 2-dimensional transverse structure behavior. For this reason, we have employed a full 3-dimensional scalar wave propagation method to simulate the performance of target designs as detailed below.

The amplitude $u$ of a propagating electric field in normal fiber and integrated optical waveguides with small index contrast, i.e. where refractive index differences are small compared to the index, is well described by the scalar Helmholtz wave equation.

$$
\nabla^{2} u+K_{0}^{2} n^{2}(x, y) u=0
$$

Here $\mathrm{n}(\mathrm{x}, \mathrm{y})$ is the refractive index which is assumed to depend on the transverse spatial coordinates $\mathrm{x}$ and $\mathrm{y}, \mathrm{K}_{0}$ is the free space wavenumber $2 \pi / \lambda$ where $\lambda$ is the vacuum wavelength. Because the index is independent of $\mathrm{z}$ (position along the ribbon fiber), such a structure supports modes whose shape is independent of $\mathrm{z}$. Such a mode $\mathrm{u}_{\mathrm{i}}(\mathrm{x}, \mathrm{y})$ propagates with a characteristic propagation constant $\beta_{i}$ ' which can be found from the eigenvalue equation,

$$
\begin{aligned}
& \nabla_{\perp}^{2} u_{i}+K_{0}^{2} n^{2}(x, y) u_{i}=\left(\beta^{\prime}\right)^{2} u_{i} \\
& \text { here } \nabla_{\perp}^{2}=\frac{\partial^{2}}{\partial x^{2}}+\frac{\partial^{2}}{\partial y^{2}} \text { is the transverse Laplacian operator. }
\end{aligned}
$$


Since (22) is linear, the general solution is a linear combination of such modes with arbitrary amplitudes, each changing its phase with propagation distance according to its own characteristic modal propagation constant.

Numerical solution of (22) can be difficult. However, a convenient simplification is to make the slowly varying envelope approximation. We assume that the amplitude $u$ varies mainly as $\exp \left(\mathrm{iK}_{\mathrm{c}} \mathrm{z}\right.$ ) where $\mathrm{K}_{\mathrm{c}}$ is a reference wavenumber (see below), i.e. we let

$$
u(x, y, z)=E(x, y, z) e^{i K_{i} z},
$$

where $\mathrm{E}$ is only a very weak function of $\mathrm{z}$. This means $\mathrm{E}$ varies little over an optical wavelength. In this case, substituting (24) into (22) and discarding the term $?^{2} \mathrm{E} / ? \mathrm{z}^{2}$ since it is small compared to $\mathrm{K}_{\mathrm{c}} ? \mathrm{E} / \mathrm{?} \mathrm{z}$, we are left with the so-called paraxial wave equation

$$
i \frac{\partial E}{\partial z}=-\frac{1}{2 K_{c}} \nabla_{\perp}^{2} E+\frac{K_{c}}{2}\left(1-\frac{n^{2}(x, y)}{n_{c}^{2}}\right) E
$$

where $n_{c}$ is the index corresponding to $K_{c}$, i.e. $K_{c}=K_{0} n_{c}$.

Note that this equation has exactly the form of the Schroedinger equation of quantum mechanics. Propagation distance $\mathrm{z}$ plays the same role in optical propagation as time does in the Schroedinger equation and the "potential energy" is given by the second right hand side term in (25) involving the refractive index. There is thus a one to one analogy between optical waveguides and 2-dimensional $(\mathrm{x}, \mathrm{y})$ quantum mechanics. We can rewrite $(25)$ as,

$$
i \frac{\partial E}{\partial z}=H E=\left(-\frac{1}{2 K_{c}} \nabla_{\perp}^{2}+\frac{K_{c}}{2}\left(1-\frac{n^{2}(x, y)}{n_{c}^{2}}\right)\right) E
$$

thus defining an optical Hamiltonian operator $\mathrm{H}$ as in quantum mechanics. The eigenvalues and eigenfunctions of this operator are the fundamental quantities that describe the nature of the optical waveguide. It can be shown that the modal eigenfunctions of Eqs. (23) and (26) are exactly the same. ${ }^{8}$ However, their eigenvalues are different. If

$$
H u_{i}=\beta_{i} u_{i}
$$

then the relationship between $\beta_{\mathrm{i}}$ and $\beta_{\mathrm{i}}{ }^{\prime}$ is

$$
\beta^{\prime}=\sqrt{K_{c}^{2}-2 K \beta} \approx K_{c}-\beta
$$

That is, $\beta$ represents the small change of the modal wavenumber $\beta$ ' from the reference wavenumber $\mathrm{K}_{\mathrm{c}}$. Because of the analogy with the Schroedinger equation, we see immediately that if we choose the cladding index to define $\mathrm{K}_{\mathrm{c}}$, then guided modes will 
have negative values of $\beta$ (corresponding to bound states in quant um mechanics having negative energy) and radiation modes will have positive values of $\beta$.

An ideal optical structure made up of repeated units is thus analogous to a crystal and is sometimes referred to as a photonic crystal. This is the type of structure we propose here. From solid state physics, we immediately understand that the modal propagation constants in such a structure will naturally be grouped into bands, and that there may be photonic band gaps in allowed propagation depending on the optical parameters.

We solve (26) numerically using the Fast Fourier Transform based Beam Propagation Method (BPM) described in the references. ${ }^{9}$ An advantage of this approach is that for a lossless/gainless medium, it exactly conserves electromagnetic energy. This is important so that reliable calculations can be carried out for a medium with small gain or loss. The solution found for (26) is of the form $\mathrm{E}(\mathrm{x}, \mathrm{y}, \mathrm{z})$ where $\mathrm{E}$ is, in general, a linear combination of modes excited at the start of the calculation by assumption of an initial field $\mathrm{E}(\mathrm{x}, \mathrm{y}, 0)$. The modal propagation constants can be found by forming a correlation function $\mathrm{P}(\mathrm{z})$

$$
P(z)=\iint E^{*}(x, y, 0) E(x, y, z) d x d y
$$

Since $\mathrm{E}$ is a linear combination of orthonormal modes, the correlation will necessarily be of the form

$$
P(z)=\sum\left|A_{n}\right|^{2} e^{i \beta_{n} z}
$$

Where $A_{n}$ is the amplitude of mode $n$ and $\left|A_{n}\right|^{2}$ is proportional to the power in mode $n$. Fourier transforming $\mathrm{P}(\mathrm{z})$ with respect to $\mathrm{z}$ yields

$\mathrm{P}(\beta)=\sum\left|A_{n}\right|^{2} \delta\left(\beta-\beta_{n}\right)$

That is, the spectrum of the correlation function consists of distinct lines centered at the modal propagation constants. Once the modal propagation constants are known, the unnormalized mode shapes can be retrieved by projecting them from the propagated field, i.e.,

$u_{n}(x, y)=\iint E(x, y, z) e^{i \beta_{n} z} d x d y$.

This technique has been used very effectively in modeling optical fibers, rib waveguides, $\mathrm{x}$ and $\mathrm{y}$ couplers and optical resonators.

The above formalism remains the same in the case of a non-passive device, i.e. a device with distributed gain or loss. In this case, the delta function lineshapes in the spectrum of (31) are broadened by an amount proportional to the imaginary part of the modal wavenumber. A numerical technique that "measures" this width, then gives a direct value for the modal gain. 


\subsection{Simulations}

We have used a numerical code embodying the above formalism in our simulations of the 2-dimensional transverse ribbon structure. Typically, the inserted field is propagated on a $256 \times 64$ grid and the propagated field evaluated as a function of transverse coordinates $\mathrm{x}$ and $y$ at 32800 longitudinal $z$ values. The complex modal propagation constants and modal field patterns can then be calculated.

A cross section of a sample ribbon laser structure is shown in Fig. 11. Both refractive index and small signal gain are spatially distributed. Simulations are started with an initial field. Part of this field projects onto waveguide modes and is propagated. The rest is radiated away from the structure. To prevent this radiated energy from reflecting from the numerical boundaries of the simulation, an absorbing layer is placed around the outer boundary.

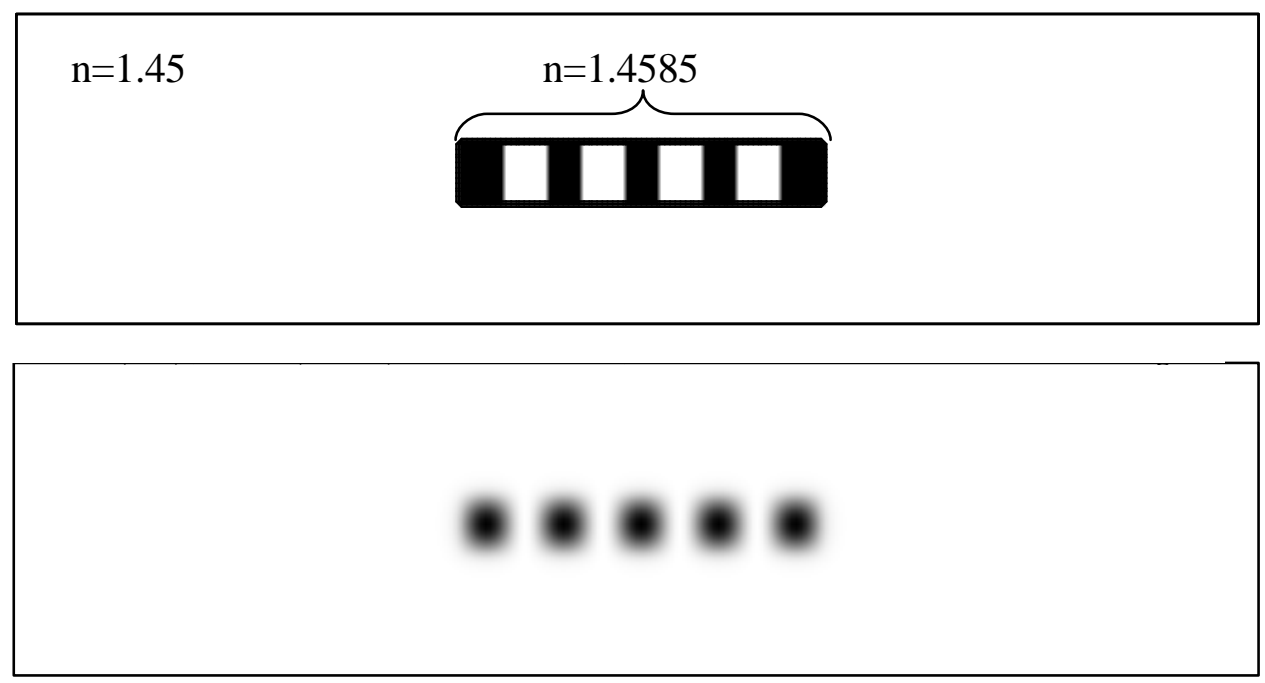

Figure 11 Cross sectional view of ribbon structure with 2 transverse dimensions that is analyzed in the text. The gain loaded portions of the waveguide are indicated by the dark regions in the upper picture. The refractive index is constant throughout the waveguide region and equals 1.4585.

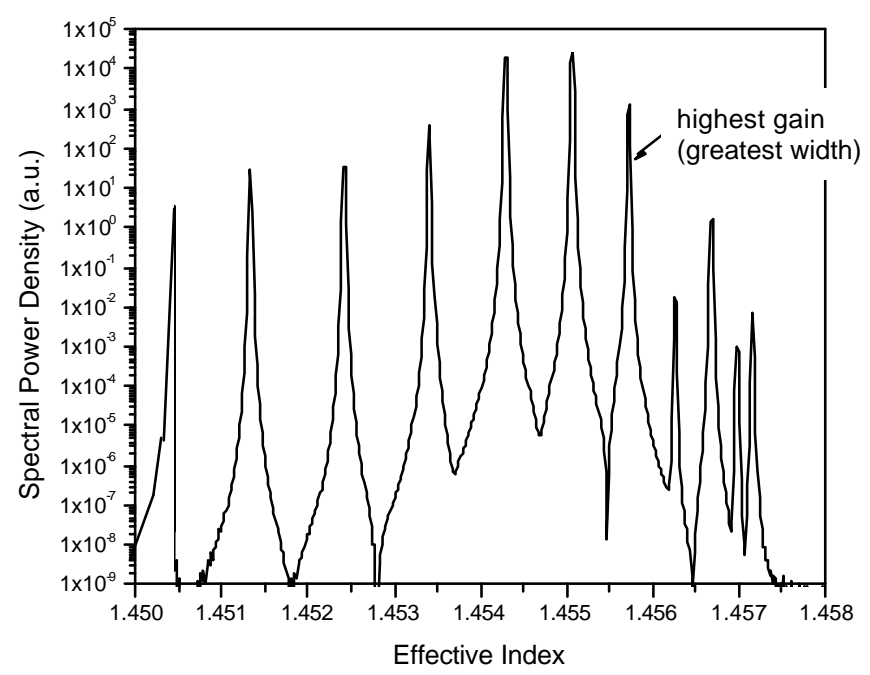

Figure 12 Spectral power of modes excited by Gaussian beam inserted into structure described in Fig. 11. 
Propagating the initial field and calculating the correlation function defined in (30) and its Fourier transform with respect to propagation distance leads to the spectrum shown in Fig.12. Excited modes appear as distinct spectral lines. Table V lists the wavevector values and effective index values of the spectral peaks in Fig. 12. For convenience of comparison we also list the wavevector and effective index values found with the 1-dimensional model for the corresponding 1-dimensional strucutre generated by by taking a line out along the $\mathrm{x}$-axis of the 2-dimensional structure depicted in Fig 11.

Table V Comparison of Modes Found Using Corresponding 1-D and 2-D Models

\begin{tabular}{|c|c|c|c|c|c|c|}
\hline $\begin{array}{c}\text { Mode } \\
\text { Number }\end{array}$ & \multicolumn{2}{|c|}{$\begin{array}{c}\text { Wavevector } \\
\left(\mu \mathbf{m}^{-1}\right)\end{array}$} & \multicolumn{2}{c|}{ Mode Effective Index } & \multicolumn{2}{c|}{ Gain Overlap } \\
\hline & \multicolumn{2}{|c|}{} & & & & \\
\hline 1 & 8.720 & 8.727 & 1.45719 & 1.45844 & 0.4154 & 0.4998 \\
\hline 2 & 8.719 & 8.726 & 1.45701 & 1.45825 & 0.3876 & 0.4989 \\
\hline 3 & 8.717 & 8.724 & 1.45671 & 1.45795 & 0.42688 & 0.4960 \\
\hline 4 & 8.714 & 8.722 & 1.45629 & 1.45751 & 0.3953 & 0.4814 \\
\hline 5 & 8.711 & 8.718 & 1.45575 & 1.45696 & 0.7436 & 0.8308 \\
\hline 6 & 8.707 & 8.714 & 1.45509 & 1.45629 & 0.46586 & 0.5543 \\
\hline 7 & 8.703 & 8.710 & 1.45432 & 1.45550 & 0.46738 & 0.5456 \\
\hline 8 & 8.697 & 8.704 & 1.45343 & 1.45459 & 0.47568 & 0.5445 \\
\hline 9 & 8.691 & 8.698 & 1.45244 & 1.45357 & 0.4739 & 0.5436 \\
\hline 10 & 8.685 & 8.691 & 1.45136 & 1.45244 & 0.39566 & 0.5394 \\
\hline 11 & 8.678 & 8.684 & 1.45022 & 1.45123 & 0.02086 & 0.5232 \\
\hline
\end{tabular}

In both the 1-D and 2-D analyses, the $5^{\text {th }}$ mode is the one with the highest gain.

\section{Robustness of designs to variations in refractive index and width dimensions}

The ultimate goal of this study is to develop an understanding of robust fiber ribbon designs that will guarantee single transverse mode operation in the presence of strong gain saturation. As such, it is useful to develop a criterion for how tightly design tolerances, both cell refractive indices and cell dimensions, must be held for the various cells that comprise a given structure. We investigate this issue in two different regimes. First we look at the impact of systematic variations in cell refractive indices and cell dimensions. This type of systematic error represents what we will likely see in structures fabricated using a fiber pulling technology. Cell dimensions will tend to vary together, shrinking below or expanding above the design point due to the manner in which the ribbons are pulled. Also, because we anticipate using the same starting material for all the gain and all the no-gain portions of the various cells that comprise the waveguide region of the ribbon, we expect index errors from the desired design point to occur uniformly throughout the structure. In addition to investigating these systematic errors, we also then go on to investigate random errors in both cell refractive indices and cell dimensions for very large, 100 core structures. Such errors characterize uncontrolled 
aspects of the ribbon structure fabrication and could be an issue in the developing technology we anticipate using.

\subsection{Systematic Errors}

Here we determine what the variations in the refractive indices of the gain and nogain regions can be and still ensure the structure will support only a single transverse mode. Because the 1-D code can be quickly executed, it is better suited to running the large number of cases required supporting this parameter study. The 2-D code was then used on a smaller scale to confirm the results of the 1-D code.

Using the constant index 5-core structure depicted in Fig. 13, a series of runs were made to determine sensitivity to systematic variations in the refractive indices of the gain and no-gain cells. In this series of runs we kept the geometry constant, allowing only the index difference to vary from run to run. The ribbon had a total of 5 gain cores, beginning and ending with gain regions. All pieces were rectangular in cross-section with the gain and no-gain regions the same height, i.e. there was no border of no-gain material surrounding the structure. The line across the middle represents the line out of the structure used in the 1-D model.

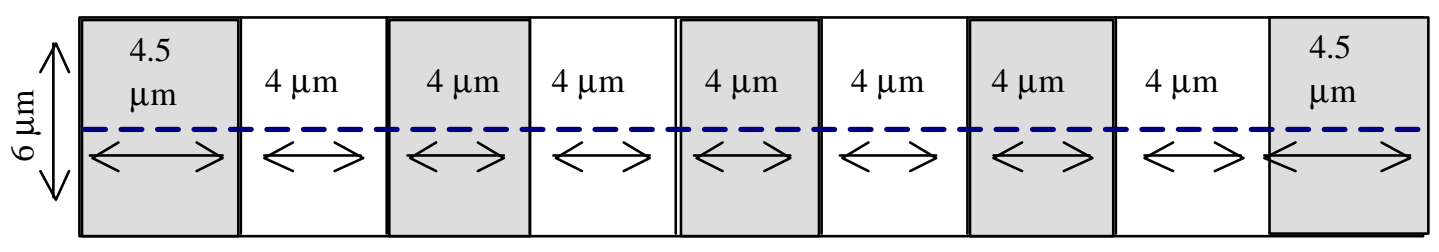

Figure 13 Structure modeled in the one dimensional and two dimensional performance sensitivity runs. This structure was held constant while the index was varied in the first series of runs and considered the constant geometry in the geometry sensitivity runs. The heavy dashed blue line represents the onedimensional line out of the structure. Shaded regions are gain-loaded.

We used an index of 1.45 for the cladding, while the gain-doped regions were held fixed at an index of 1.4585 and the non-gain regions were allowed to vary between 1.4628 and 1.4542. The data were compiled by the delta index value of "gain index - no-gain index". This range of allowed indices for the no-gain regions represents both "guidedlike" (gain index > non-gain index) and "anti- guided-like" (gain index < non-gain index) variations. For each index difference, the largest and second largest gain overlap modes were recorded. A total of 11 cases were run using the one-dimensional model. Five of the 11 cases were rerun using the two dimensional model. Figure 14 is a plot of Overlap vs. Index Difference and presents both the 1-D and 2-D results. 
Table V Overlap of two strongest eigenmodes of 1-dimensional structure with varying index differences

\begin{tabular}{|c|l|l|l|l|l|l|}
\hline \multirow{2}{*}{ Delta Index } & \multicolumn{3}{|l|}{ Highest Gain Overlap Mode } & \multicolumn{2}{l|}{$\begin{array}{l}\text { 2nd Highest Gain Overlap } \\
\text { Mode }\end{array}$} \\
\cline { 2 - 7 } & $\begin{array}{c}\text { Mode } \beta \\
(\mathbf{1} / \mu \mathbf{m})\end{array}$ & $\begin{array}{l}\text { Effective } \\
\text { Index }\end{array}$ & $\begin{array}{c}\text { Overlap } \\
\Gamma\end{array}$ & $\begin{array}{l}\text { Mode } \beta \\
(\mathbf{1} / \mu \mathbf{m})\end{array}$ & $\begin{array}{l}\text { Effective } \\
\text { Index }\end{array}$ & $\begin{array}{c}\text { Overlap } \\
\Gamma\end{array}$ \\
\hline 0.0043 & 8.7153 & 1.4564 & 0.88 & 8.7160 & 1.4566 & 0.87 \\
0.0033 & 8.7159 & 1.4565 & 0.88 & 8.7167 & 1.4567 & 0.86 \\
0.0023 & 8.7166 & 1.4567 & 0.87 & 8.7175 & 1.4568 & 0.82 \\
0.0013 & 8.7173 & 1.4568 & 0.86 & 8.7188 & 1.4570 & 0.73 \\
0.0003 & 8.7182 & 1.4569 & 0.84 & 8.7209 & 1.4574 & 0.55 \\
0.0000 & 8.7184 & 1.4570 & 0.84 & 8.7144 & 1.4563 & 0.56 \\
-0.0003 & 8.7187 & 1.4570 & 0.83 & 8.7151 & 1.4564 & 0.61 \\
-0.0013 & 8.7198 & 1.4572 & 0.81 & 8.7171 & 1.4567 & 0.71 \\
-0.0023 & 8.7210 & 1.4574 & 0.78 & 8.7186 & 1.4570 & 0.74 \\
-0.0033 & 8.7224 & 1.4576 & 0.75 & 8.7202 & 1.4572 & 0.74 \\
-0.0043 & 8.7240 & 1.4579 & 0.72 & 8.7218 & 1.4575 & 0.71 \\
& & & & & & \\
\hline
\end{tabular}

Table VI Overlap of two strongest eigenmodes of 2-dimensional structure with varying index differences

\begin{tabular}{|c|l|l|l|l|l|l|}
\hline \multirow{2}{*}{ Delta Index } & \multicolumn{3}{|l|}{ Highest Gain Overlap Mode } & \multicolumn{2}{l|}{$\begin{array}{l}\text { 2nd Highest Gain Overlap } \\
\text { Mode }\end{array}$} \\
\cline { 2 - 7 } & $\begin{array}{c}\text { Mode } \beta \\
(\mathbf{1} / \mu \mathbf{m})\end{array}$ & $\begin{array}{l}\text { Effective } \\
\text { Index }\end{array}$ & $\begin{array}{c}\text { Overlap } \\
\Gamma\end{array}$ & $\begin{array}{c}\text { Mode } \beta \\
(\mathbf{1} / \mu \mathbf{m})\end{array}$ & $\begin{array}{l}\text { Effective } \\
\text { Index }\end{array}$ & $\begin{array}{c}\text { Overlap } \\
\Gamma\end{array}$ \\
\hline 0.0043 & 8.7100 & 1.4555 & 0.72 & 8.7090 & 1.4554 & 0.67 \\
0.0013 & 8.7098 & 1.4555 & 0.75 & 8.7134 & 1.4561 & 0.55 \\
0.0000 & 8.7112 & 1.4557 & 0.70 & 8.7026 & 1.4543 & 0.42 \\
-0.0013 & 8.7128 & 1.4560 & 0.69 & 8.7058 & 1.4549 & 0.53 \\
-0.0043 & 8.7001 & 1.4539 & 0.61 & 8.7182 & 1.4569 & 0.57 \\
& & & & & & \\
\hline
\end{tabular}




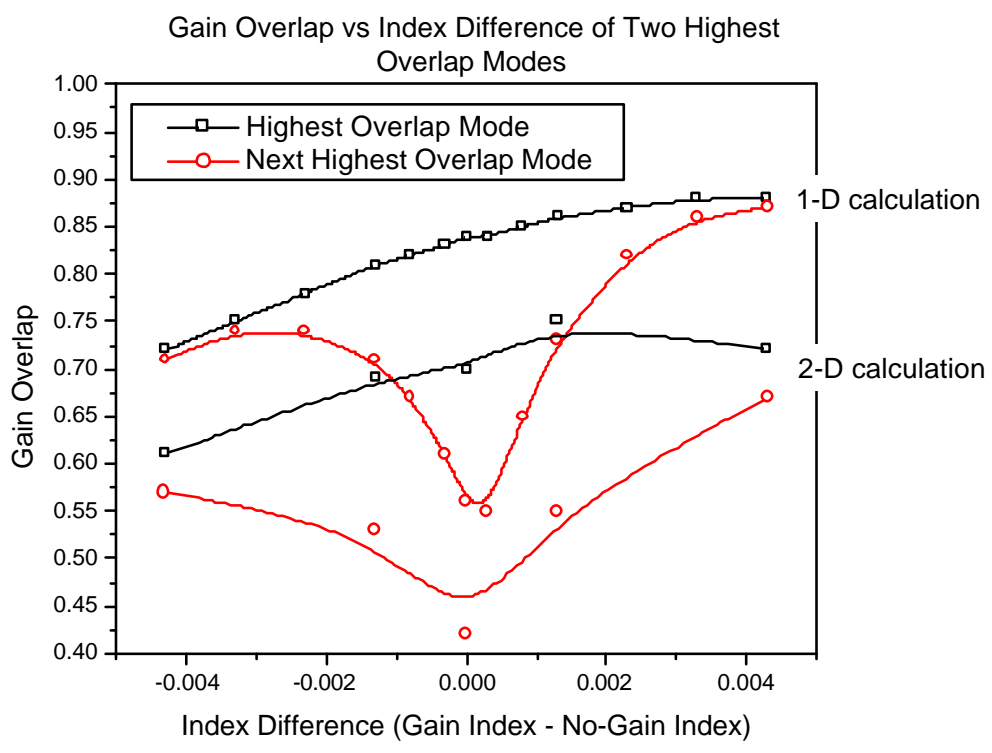

Figure 14 Overlap vs. Index Difference plot for 1-D and 2-D cases with systematic index variation between the gain and no-gain regions.

Although the gain overlap increases as the index difference increases, Figure 14 shows the point of maximum discrimination occurs with an index difference of zero. Also, evident in Fig. 14 is the qualitative similarity between the 1-D and 2-D calculations, both showing maximum gain discrimination for an index difference of 0.0. Remember, one of the critical design requirements is the structure oscillate in a single transverse mode making good mode discrimination a strong figure of merit in the design. As the index difference increases (in both the positive and negative direction), the overlap values of the two strongest modes get closer, increasing the chance the structure will "hop" from one mode to the other during operation.

We next explored the structure's sensitivity to geometry (cell width) variations. The dimensions of all cells within the structure were varied by the same amount, mimicking the type of systematic error that might be expected in fabricating the structure using a fiber pulling technique. For this parameter study we varied each cell width by a specified amount $( \pm 2 \%, \pm 4 \%, \pm 6 \%, \pm 8 \%$, and $\pm 10 \%)$ from its original value. This parameter study was repeated for systematic index variations of $-0.0013,0.0$, and 0.0013 . All runs were done with the 1-D model. As seen in Figure 15 where the results of this study are plotted, varying the cell widths has little impact on either the gain overlap value or the mode discrimination out to the $\pm 10 \%$ systematic variations investigated here. 


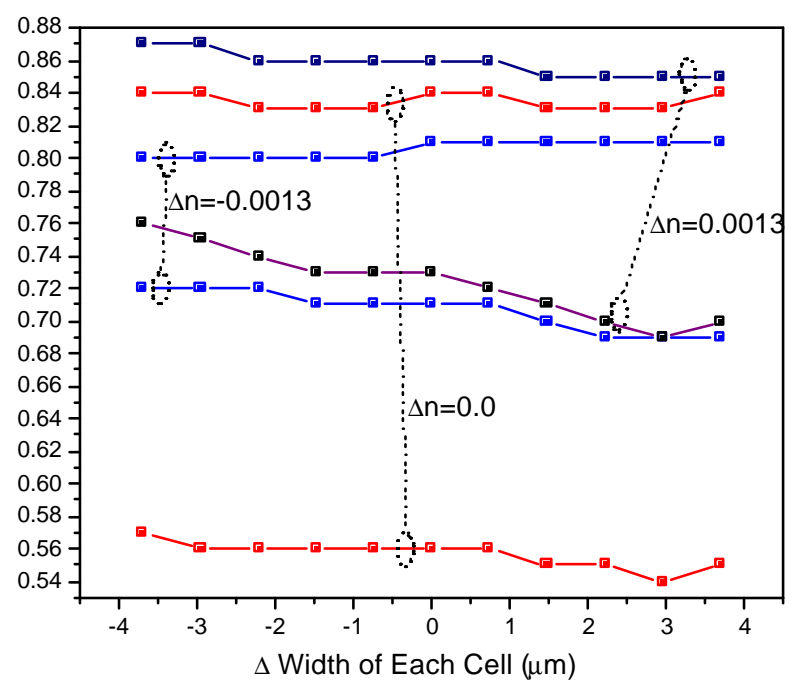

Figure 15 Gain Overlap vs. Index Difference of the two highest overlap modes for three different values of cell $\Delta n$. All data points were calculated using the one-dimensional model.

Based on these results, we feel an acceptable index difference is \pm 0.001 with a target difference of 0.0. Within this index difference range, we should be able to handle cell width fabrication errors of $\pm 10 \%$ and possibly more.

Important for power scaling is the question of required index tolerance as a function of the number of cores. To answer this question, we have repeated the forgoing 5 -core structure analysis in which the refractive index of the gain and no-gain cells was systematically varied for a 20 core and 100 core ribbon fiber structure. Figure 16 depicts the results of this study. The data in Fig. 16 was generated using the 1-D code with subcell dimensions identical to those depicted in Fig. 13. Evident in Fig. 16 is the observation that, as the number of cells increases the requirements on the systematic variation of the refractive index become more stringent. To better quantify this scaling law, we have determined the FWHM spread in index for which the gain discrimination between the two highest overlap modes just halves from its peak value at $\Delta \mathrm{n}=0$. These FWHM values are indicated in Fig. 16. In Fig. 17, we plot these FWHM index spreads against the inverse number of gain cores $\left(1 / n_{\text {cores }}\right)$ for the three cases studied here. As seen from the data plot in Fig. 17, the $\Delta \mathrm{n}_{\mathrm{FWHM}}$ requirement varies almost linearly with $1 /$ $\mathrm{n}_{\text {cores }}$, which is what we intuitively expect. However, a variation of $\Delta \mathrm{n}_{\text {FWHM }}$ as (1/ $\left.\mathrm{n}_{\text {cores }}\right)^{0.75}$ gives a better empirical fit to the data than does a variation as $1 / \mathrm{n}_{\text {cores. }}$. The solid line in Fig. 17 is a plot of $0.0084\left(1 / \mathrm{n}_{\text {cores }}\right)^{0.75}$, which represents the best least squares fit of this empirical functional form to the data. 


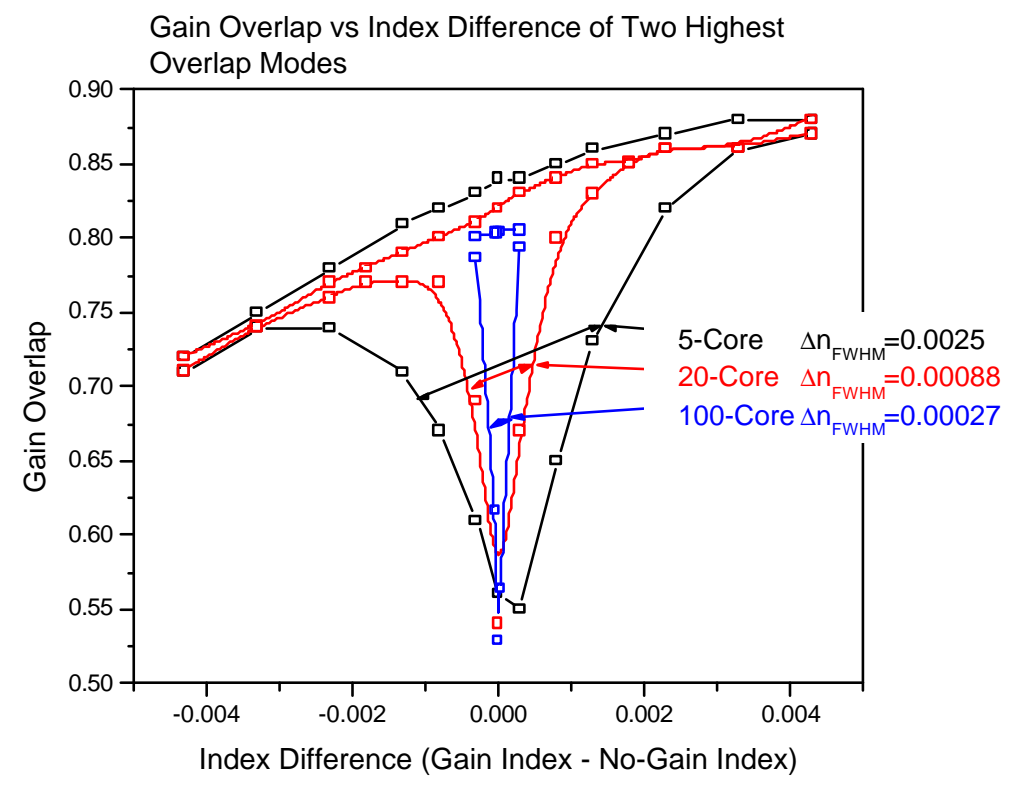

Figure 16 Overlap vs. Index Difference plot for 1-D structures having a systematic index variation between the gain and no-gain regions. Three different structures were investigated consisting of 5, 20 and 100 cores, respectively. For each structure investigated the gain overlap of the highest overlap and next highest overlap mode are plotted against the systematic index variation.

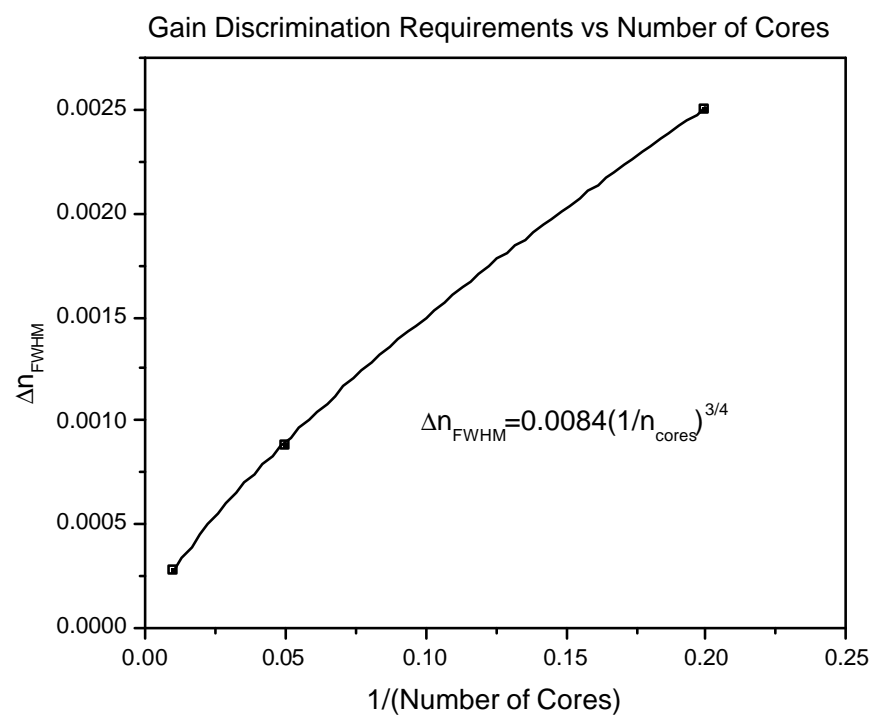

Figure 17 Gain discrimination requirements vs. the number of mode cores taken from the data in Fig. 16. The FWHM spread in index plotted here is defined by the two points at which the gain discrimination between the two highest overlap modes just halves from its peak value at $\Delta n=0$. The curve is represents a least squares fit of the functional form displayed to the data. 


\subsection{Random Errors}

In addition to the systematic errors just considered, random variations in cell index and cell dimension may be an issue, particularly in fiber structures consisting of a large number of cells. To investigate the impact of random index variations we have modeled a 100-core ribbon fiber with nominally constant index throughout the waveguiding region. The particular 1-dimensional waveguide design investigated here consists of a structure similar to the one of Fig. 13 but with cell dimensions of $3.65 \mu \mathrm{m}$ for the central cells and $4.36 \mu \mathrm{m}$ for the 2 end cells. The impact of random variations in cell refractive index is illustrated in Fig. 18 where we have plotted the structure's eigenmode gain overlap against the various mode effective refractive indices. In each case, the individual cells that comprise the waveguide have had their refractive indices randomly varied with a uniform spread about the design point. The magnitudes of the uniform random distributions were taken to be $\Delta \mathrm{n}=0, \Delta \mathrm{n}= \pm 0.00015, \Delta \mathrm{n}= \pm 0.00037$, and $\Delta \mathrm{n}= \pm 0.00073$ as indicated. In Fig. 19, the overlaps of the two highest gain overlap modes for this structure are plotted as a function of the random error introduced in the individual cell refractive indices. Examining this plot, it is seen that the gain discrimination between the two highest overlap modes begins to substantially degrade when random index variations are approximately $\Delta \mathrm{n}= \pm 0.00037$, in approximate accord with the FWHM deduced in Figs. 16 and 17. We have also modeled the impact of random variations in the cell dimensions for the 100-core structure studied here and found in general it is very robust to these types of variations. Based on our modeling, random dimensional variations at the $\pm 10 \%$ level do not significantly degrade the mode overlap and mode overlap discrimination and so are probably not an issue with the device fabrication. 


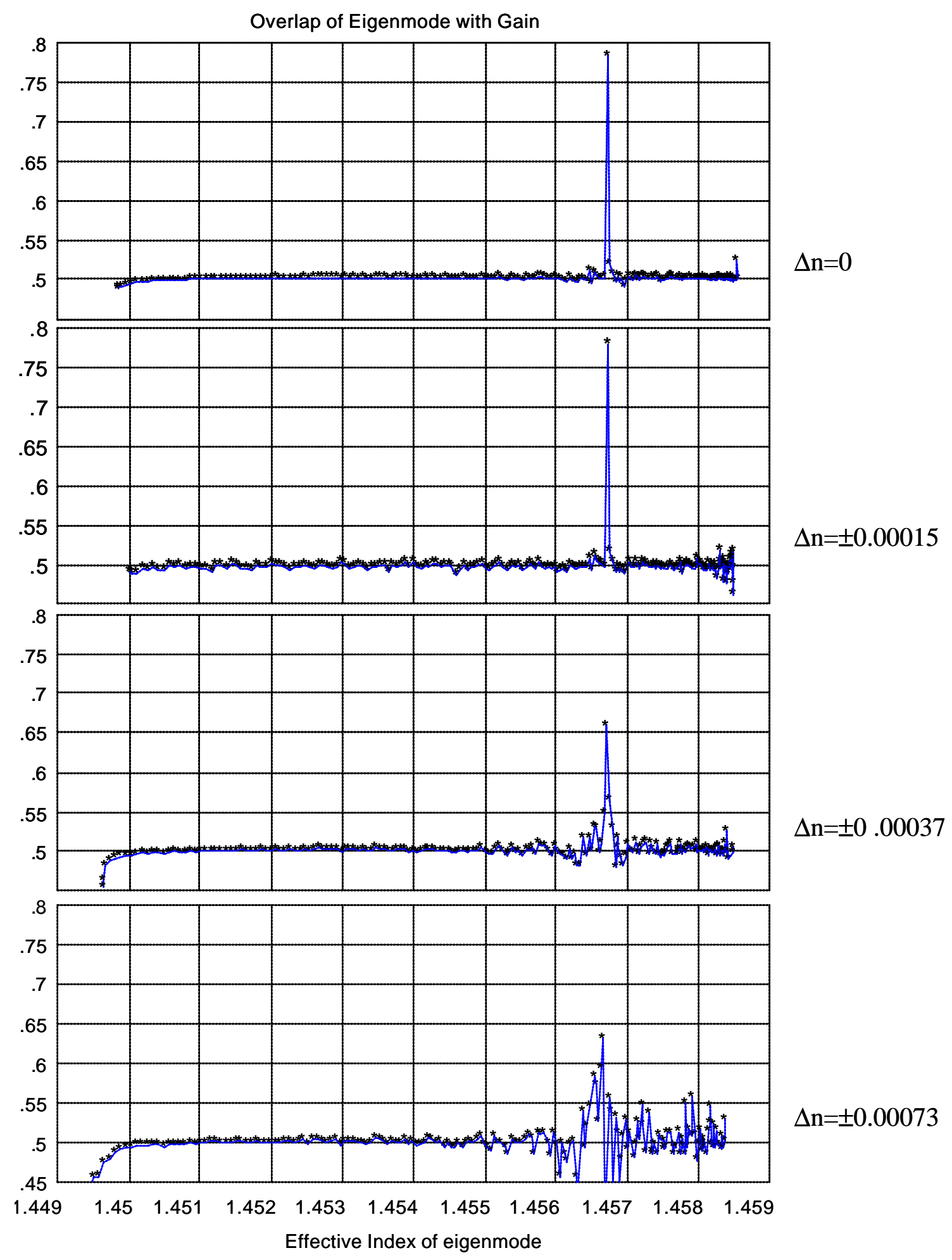

Figure 18 The impact of random variations in cell refractive index are illustrated here using a 100 core structure in which gain overlap of the structure's eigenmodes are plotted against the various mode's effective refractive index. In each case, the individual cells that comprise the waveguide have had their refractive indices randomly varied with a uniform spread about the design point. The magnitudes of the uniform random distributions were taken to be $\Delta \mathrm{n}=0, \Delta \mathrm{n}= \pm 0.00015, \Delta \mathrm{n}= \pm 0.00037$, and $\Delta \mathrm{n}= \pm 0.00073$ as indicated. 


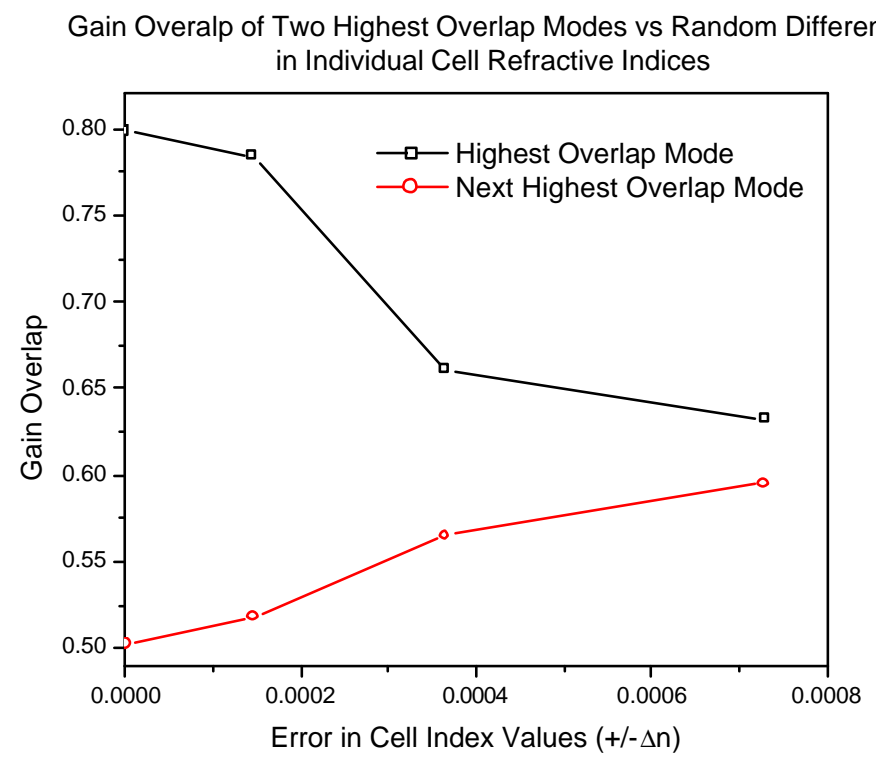

Figure 19 Gain overlap for 100-core ribbon fiber. The overlap of the two highest gain overlap modes is plotted as a function of the random error introduced in the individual cell refractive indices. Random errors in refractive indices are uniformly distributed with the indicted amplitudes.

\section{Pump Coupling and Enegetics Performance}

The proposed ribbon laser structures we have been discussing within would use a side-pumping scheme, with pumps placed periodically along the fiber. Figure 20 shows the way a nominally $50 \mathrm{~W}$ bar is coupled to the pump cladding via a diffraction grating. Note that other than this grating, there is no beam conditioning of the diode, making the pump module simple. This is an important advantage of our proposed approach over other more conventional end-pumped approaches, as the diode lasers used in the side pumping approach require no exotic or expensive radiance conditioning.

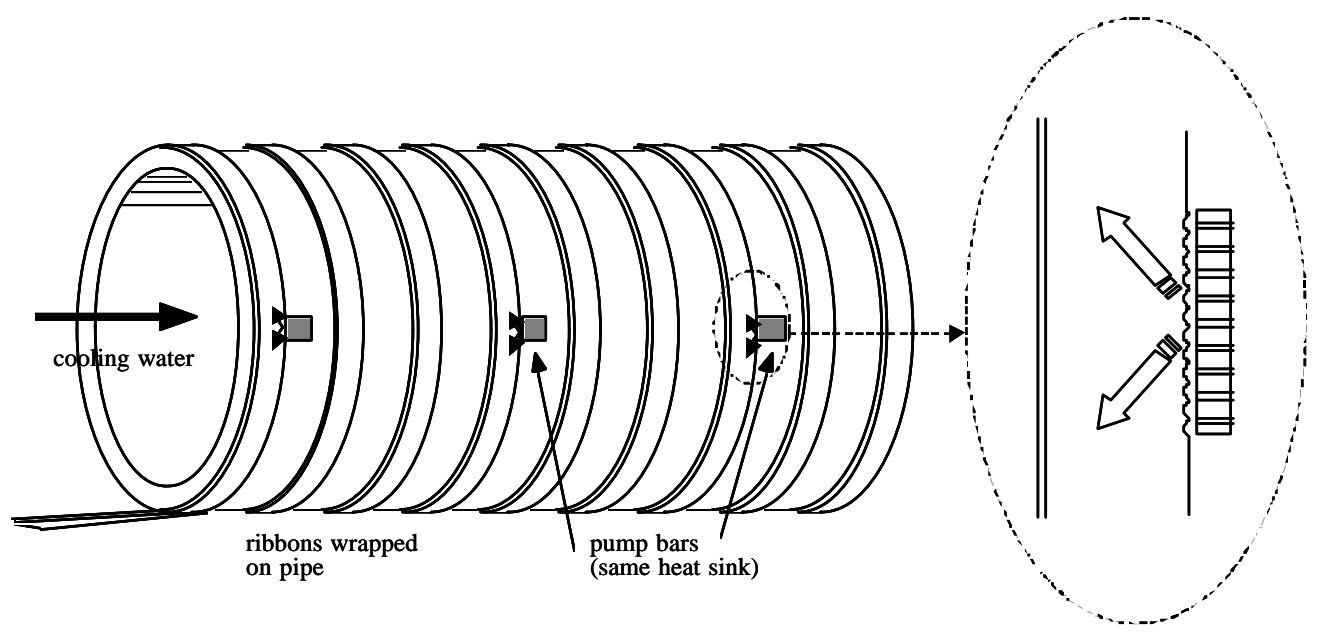

Figure 20 Diode bar side-pumping scheme. 
As a preliminary step to demonstrating a large multiple core device, we propose first demonstrating a 5-core structure. The goal of this first prototype device is to convincingly demonstrate that we can operate in a single transverse mode that exhibits good intensity uniformity across the entire array of gain cores. To take advantage of the large body of work that has already been developed toward the fabrication of holey fibers, we are initially proposing that our ribbon structure will be fabricated using the same technology 10 . In particular, we propose collaborating with Prof. Phillip Russell and his group at the University of Bath to fabricate the 5-core Nd:Silica structure depicted in Fig. 21.

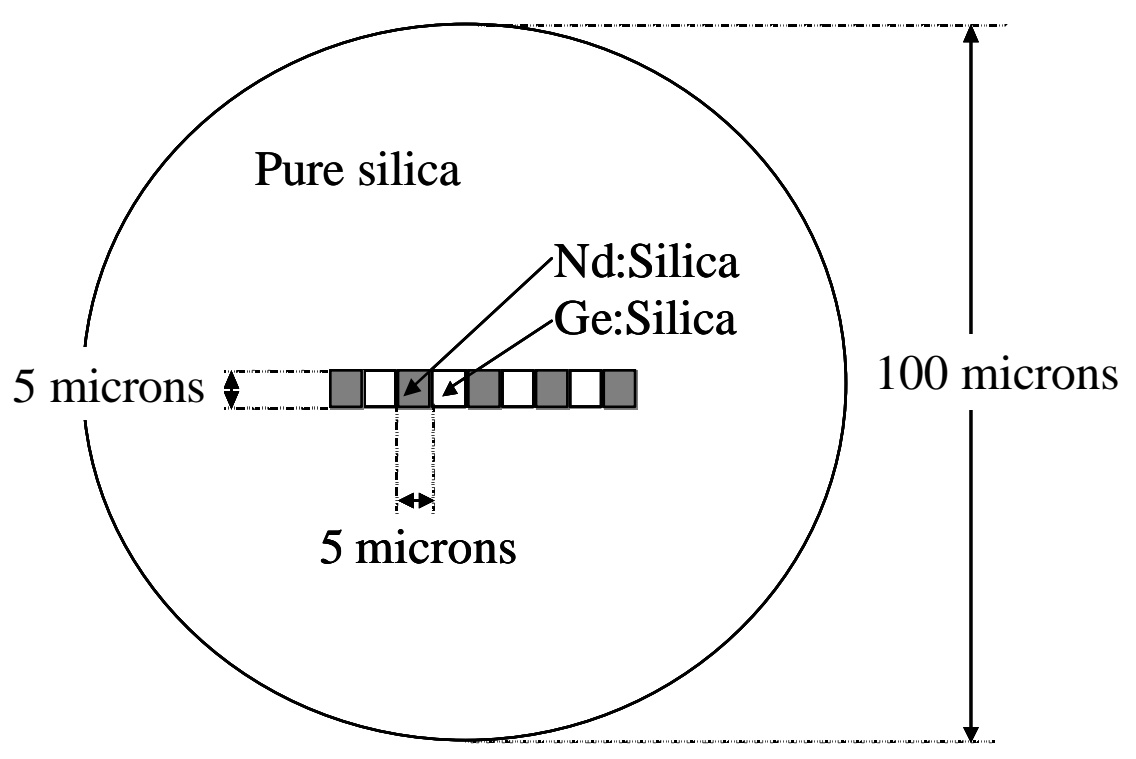

Figure 21 Using the same technology currently used to fabricate holey fibers, we are collaborating with the University of Bath to have the pictured prototype 5-core device fabricated.

The choice of going with a round cross-sectioned pump cladding region is to stay compatible with the presently developed holey fiber technology, although we ultimately are interested in going to rectangular cross-sectioned pump cladding to be compatible with our proposed side-pumping scheme shown in Fig. 20. We anticipate doing initial laser mode characterizations with the structure shown in Fig. 21 configured as an oscillator. The proposed silica based design would use a fiber having a length of $25 \mathrm{~m}$ and end-pumped by an approximately $1 \mathrm{~W}$ source. The refractive index of the waveguiding region is nominally designed to be constant in value and larger than the surrounding pump cladding by 0.0085 . The gain cores are doped with $\mathrm{Nd}$ at $1 \times 10^{19} / \mathrm{cm}^{3}$. A simple energetics model ${ }^{11}$ has been adapted to this structure and predicts $270 \mathrm{~mW}$ of generated laser output power, or roughly a $27 \%$ opticaloptical conversion efficiency. Figure 22 is a plot of model predicted output power against output coupler reflectivity showing optimum performance with a $15 \%$ reflective output coupler. 


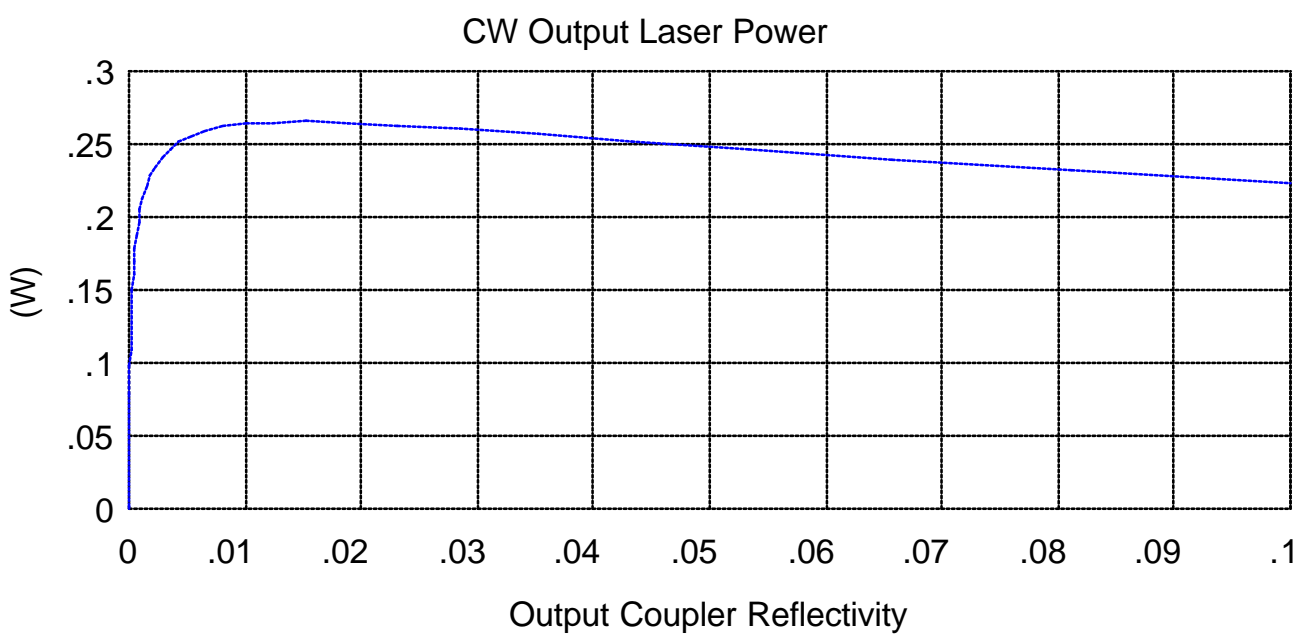

Figure 22 Predicted 5-core prototype ribbon fiber energetics performance.

The mode overlap as a function of the effective refractive index, and the intensity profile of the favored high overlap mode follow in Figs. 23 and 24, respectively.

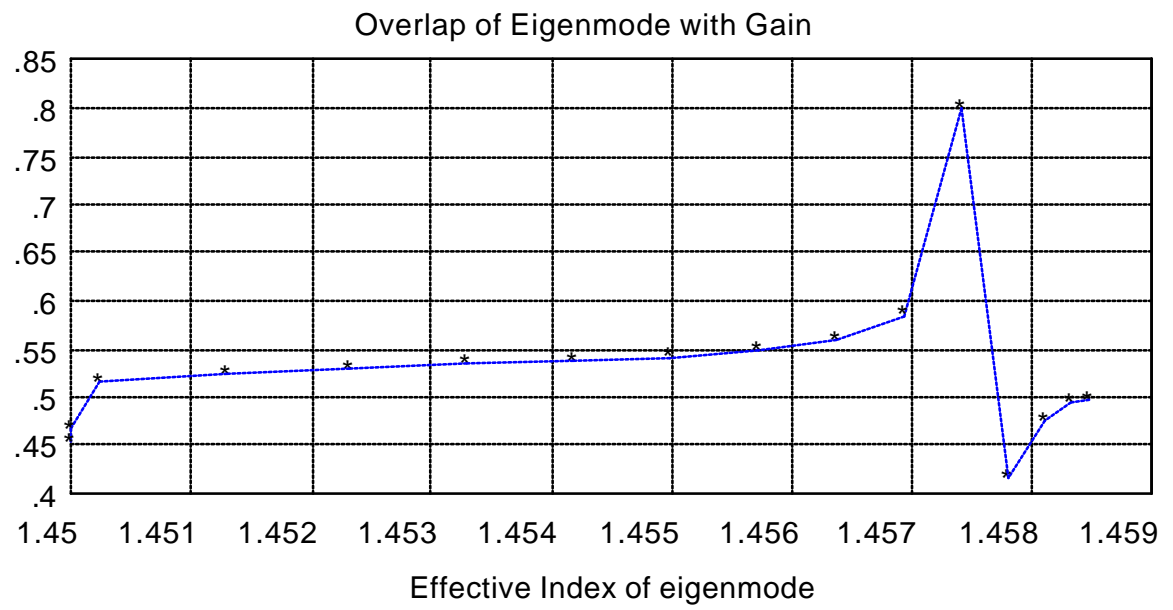

Figure 23 Mode overlap with gain region for prototype 5-core structure as a function of mode refractive index (1-dimensional calculation).

Eigenmode Intensity Profile \#5

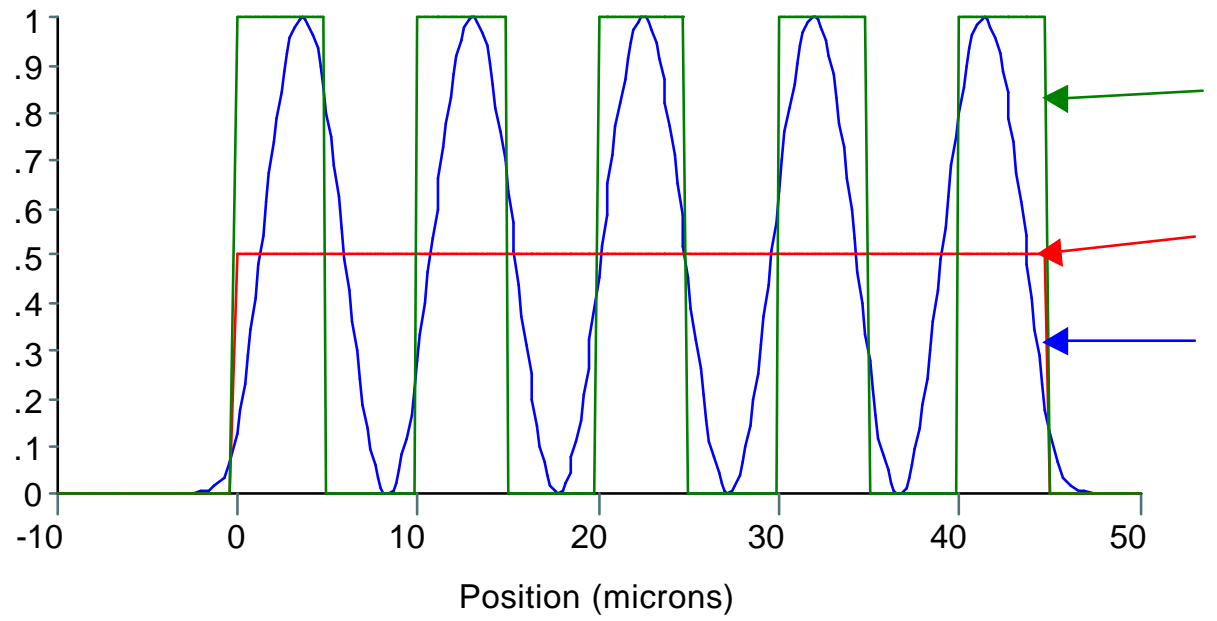

Gain regions $(1=$ gain and $0=$ no-gain)

Refractive Index (not to scale)

Intensity of highest overlap mode

Figure 24 Intensity profile of highest gain overlap mode. 
${ }^{1}$ T.M. Monro, D.J. Richardson, N.G.R. Broderick, and P.J. Bennet, "Holey Optical Fibers: An Efficient Modal Model," Journal of LightWave Technology, 17, p. 1093 , 1999.

${ }^{2}$ V. Dominic, S. MacCormack, R. Waarts, S. Sanders, S. Bricknese, R. Dohle, E. Wolak, P.S. Yeh, and E. Zucker, “110 W Fiber Laser," CLEO '99 Postdeadline Papers CPD11-1.

${ }^{3}$ P. Glas, M. Naumann, A. Schirrmacher, Th. Pertsch, "A cw diode-pumped single-silica fiber comprising 40 cores used as active elements for a high power fiber laser at $1050 \mathrm{~nm}$," CLEO '98 Technical Digest, paper CtuK5, p 113,Optical Society of America.

${ }^{4}$ D. Meyhuys, K. Mitsunaga, L. Eng, W.K. Marshall, and A. Yariv, "Supermode control in diffractioncoupled semiconductor laserr arrays," Appl. Phys. Lett. 53, p. 1165, 1988.

${ }^{5}$ Photonics Spectra October 2000, see report on Peter K. Cheo's evanescently coupled 7-core structure on page 20. This work is to be published in an upcoming Photonics Technology Letters article.

${ }^{6}$ D. Botez and D. R. Scifres, Diode Laser Arrays, Cambridge Univ. Press 1994.

${ }^{7}$ T.A. Birks, J.C. Knight, and P.St. Russell, “Endlesly single-mode photonic crystal fiber,” Opt. Lett. 22, p 961 (1997).

${ }^{8}$ M.D. Feit and J.A. Fleck, Jr., "A Spectral Approach to Optical Resonator Theory," Applied Optics 20, 2843-51 (15 August 1981).

${ }^{9}$ M.D. Feit and J.A. Fleck,Jr., "Mode properties of optical fibers with lossy components by the propagating beam theory", Appl. Opt. 20, 848-56 (1 March 1981).

${ }^{10}$ W.J. Wadsworth, J.C. Knight, W.H. Reeves, P.St.J. Russell and J. Arriaga, "Yb ${ }^{3+}$-doped photonic crystal fibre laser," Electronic Letters, Vol. 36, No. 17 (2000).

${ }^{11}$ R.J. Beach "CW theory of quasi-three level end-pumped laser oscillators," Opt. Comm. 123, p 385 (1995). 